\title{
The mouse autonomic nervous system modulates inflammation and epithelial renewal after corneal abrasion through the activation of distinct local macrophages
}

Yunxia Xue ${ }^{1}$, Jingxin $\mathrm{He}^{1,2}$, Chengju Xiao ${ }^{1}$, Yonglong Guo ${ }^{1}$, Ting Fu ${ }^{1}$, Jun Liu ${ }^{1}$, Cuipei Lin ${ }^{1,2}$, Mingjuan Wu ${ }^{1}$, Yabing Yang ${ }^{1}$, Dong Dong ${ }^{1}$, Hongwei Pan ${ }^{1}$, Chaoyong $\mathrm{Xia}^{3}$, $\mathrm{Li} \mathrm{Ren}^{4}$ and Zhijie $\mathrm{Li}^{1,5,6}$

Inflammation and reepithelialization after corneal abrasion are critical for the rapid restoration of vision and the prevention of microbial infections. However, the endogenous regulatory mechanisms are not completely understood. Here we report that the manipulation of autonomic nervous system (ANS) regulates the inflammation and healing processes. The activation of sympathetic nerves inhibited reepithelialization after corneal abrasion but increased the influx of neutrophils and the release of inflammatory cytokines. Conversely, the activation of parasympathetic nerves promoted reepithelialization and inhibited the influx of neutrophils and the release of inflammatory cytokines. Furthermore, we observed that $\mathrm{CD} 64^{+} \mathrm{CCR} 2^{+}$macrophages in the cornea preferentially expressed the $\beta-2$ adrenergic receptor (AR), whereas CD64 ${ }^{+} \mathrm{CCR} 2^{-}$macrophages preferentially expressed the $a-7$ nicotinic acetylcholine receptor ( $\mathrm{a} 7 \mathrm{nAChR}$ ). After abrasion, the topical administration of a $32 \mathrm{AR}$ agonist further enhanced the expression of the proinflammatory genes in the $\mathrm{CD}_{64}{ }^{+} \mathrm{CCR} 2^{+}$cell subset sorted from injured corneas. In contrast, the topical administration of an a7nAChR agonist further enhanced the expression of the anti-inflammatory genes in the $\mathrm{CD}_{64}{ }^{+} \mathrm{CCR} 2{ }^{-}$subset. Thus crosstalk between the ANS and local macrophage populations is necessary for the progress of corneal wound repair. Manipulation of ANS inputs to the wounded cornea may represent an alternative approach to the treatment of impaired wound healing.

Mucosal Immunology (2018) 11:1496-1511; https://doi.org/10.1038/s41385-018-0031-6

\section{INTRODUCTION}

Corneal abrasions, which are nonpenetrating injuries to the cornea, are the most common ocular injuries in all age groups and can occur in any situation. ${ }^{1}$ Eye-related diagnoses represent approximately $13 \%$ of all emergency room visits. ${ }^{2}$ Of those, approximately $45 \%$ are corneal abrasions. If defects do not heal properly or are not treated promptly, corneal abrasions can progress to corneal ulcers, erosion, and microbial infections that result in further ocular damage and even long-term vision problems, including corneal perforation or scarring.,4 Patients who are malnourished or who have compromised corneas are particularly at risk. Consequently, a more comprehensive understanding of the underlying processes and mechanisms at work during the healing of a corneal abrasion is necessary for the efficient and specific treatment of corneal impairment.

Corneal wound closure is orchestrated by a series of events involving reepithelialization, cell division, inflammation, and remodeling. $^{5-10}$ After corneal abrasion, injured epithelial cells and keratocytes rapidly produce proinflammatory cytokines, such as interleukin (IL)-1, keratinocyte chemoattractant, LIX (a murine neutrophil-chemoattractant chemokine), macrophage inflammatory protein $1 \mathrm{a}$ (MIP-1a), tumor necrosis factor (TNF)- $\mathrm{a}$, IL-6, ${ }^{11} \mathrm{IL}-17,{ }^{6} \mathrm{IL}-20,{ }^{12} \mathrm{IL}-22{ }^{10} \mathrm{IL}-25$, and IL-33. ${ }^{9}$ These cytokines recruit immune cells into the wound area and influence reepithelialization by different mechanisms. ${ }^{5}$ Neutrophils are the first immune cell type to respond and massively populate the site of the corneal abrasion, where they release proteolytic enzymes and growth factors that help clear matrix debris and support nerve regrowth in the cornea. ${ }^{6}$ After the initial neutrophil influx, there is an influx of macrophages that maintain or dampen the local inflammation, depending on whether proinflammatory cytokines (e.g., TNF-a and IL-6) or antiinflammatory cytokines (e.g., IL-4 and IL-10) are released. ${ }^{9}$ Although inflammation can drive regeneration, it can also inhibit the restorative process if the reaction is excessive. ${ }^{7}$ Finally, at the time of inflammation, the defect area will be recovered by the remaining reepithelialization-derived epithelial layer and dividing cells.

Corneal wound repair is different from the repair of other tissues owing to several unique features. ${ }^{13}$ First, the absence of blood and lymphatic vessels limits the relative number of inflammatory cells trafficking to the wound after a corneal injury.

\footnotetext{
${ }^{1}$ International Ocular Surface Research Center, Institute of Ophthalmology, Key Laboratory for Regenerative Medicine, Jinan University Medical School, Guangzhou, China;

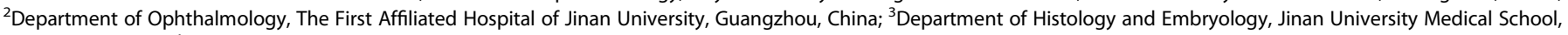

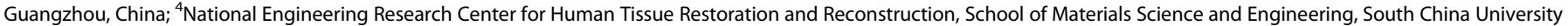

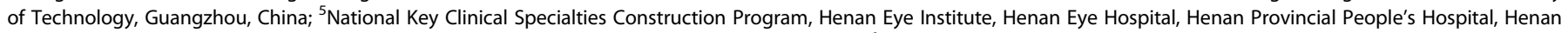

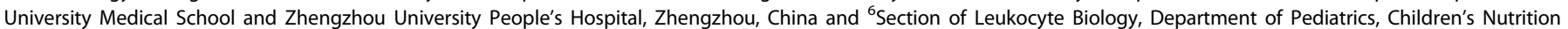
Research Center, Baylor College of Medicine, Houston, Texas, USA

Correspondence: Zhijie Li (zhijielee@yahoo.com)
}

Received: 17 December 2017 Revised: 2 April 2018 Accepted: 5 April 2018

Published online: 9 July 2018 
Second, the cornea has the highest density of nerve fibers among the peripheral tissues. This innervation plays an important role in maintaining the homeostasis of the corneal epithelium by releasing nutrients and growth factors. ${ }^{14,15}$ Third, recent observations by our study group have shown that classical natural killer cells that migrate to the cornea after corneal abrasion have newly discovered features that downregulate corneal inflammation. ${ }^{8}$ However, a dysfunction or imbalance in such protective mechanisms may lead to abnormal inflammatory responses and permanent tissue impairment after an injury. Thus exploring the detailed regulatory mechanism of wound repair and inflammation after abrasion will be beneficial for the development of new therapeutic strategies that accelerate wound repair without unwanted side effects.

The autonomic nervous system (ANS), a division of the peripheral nervous system, is involved in the tissue regeneration process. The ANS exists in different tissues and organs. Autonomic nerves are present in all mammalian corneas, although the density varies among species. ${ }^{16}$ In rabbit and cat corneas, sympathetic fibers account for $10-15 \%$ of nerve fibers, whereas in primates (including humans), using the similar techniques, sympathetic fibers are rarely found or are absent. ${ }^{15,17}$ Parasympathetic nerves also exist in the corneas of different species, with varied densities in rats, cats, dogs, and humans. ${ }^{15,18}$ However, it should be noted that the neuroanatomy of the ANS plexus innervating the murine cornea remains incompletely characterized. ${ }^{19}$ The ANS balances the physiological functions of these tissues and organs through two branches, the sympathetic and parasympathetic nervous systems (SNS and PSNS, respectively). In neonatal mice, the mechanical interruption of the left vagus nerve, which is the predominate neural effector of the PSNS, was found to suppress regenerative responses in the heart following injury. ${ }^{20}$ Moreover, mice subjected to sympathectomy exhibit failed cardiac regeneration following apical resection surgery. ${ }^{21}$ Thus, in principle, the wound repair of the mammalian cornea could also be regulated by the ANS.

The SNS highly innervates both primary and secondary lymphoid tissues, and its nerve terminals lie in close proximity to the immune cells. ${ }^{22}$ Thus the activation of the SNS can directly regulate the immune system by releasing the predominate adrenergic neurotransmitters epinephrine and norepinephrine. Additionally, immune cells, including macrophages, express $\alpha / \beta$-adrenergic receptors (ARs) on the cell membrane. ${ }^{23}$ These receptors allow immune cells to respond to neurotransmitters such as catecholamines (CChs: norepinephrine, epinephrine, and dopamine) released by the SNS nerve endings. Recent studies have further suggested a complex role of the SNS in regulating the immune system by crosstalk with local macrophages. ${ }^{24}$ For example, a subset of macrophages in the intestine and adipose tissue not only preferentially express $\beta 2 A R s$ but are also innervated by norepinephrine-secreting enteric neurons and sympathetic fibers. ${ }^{25,26}$ The communication between these neurons and macrophages rapidly induces tissue-protective responses to distal perturbations such as bacterial infections and transforms these macrophages into their proinflammatory state. Another recent landmark study found that the catecholaminergic splenic nerve can stimulate a subset of $\mathrm{CD}^{+}{ }^{+} \mathrm{T}$ memory cells in the spleen to release acetylcholine $(\mathrm{ACh}){ }^{27}$ These $\mathrm{CD}^{+}{ }^{+} \mathrm{T}$ cells perform a key regulatory role in the prevention of excessive inflammation through the activation of the $a-7$ nicotinic acetylcholine receptor ( $\mathrm{a} 7 \mathrm{nAChR})$ expressed on splenic macrophages.

The PSNS also continuously monitors and responds to inflammatory stimuli through its cholinergic anti-inflammatory pathways. ${ }^{28}$ The anti-inflammatory response is triggered of ACh as signals transmitted by the PSNS to the periphery, mainly by way of ACh. ${ }^{29,30}$ These ACh molecules act on muscarinic and nicotinic $A C h$ receptors ( $m A C h R s$ and $n A C h R s$, respectively) on immune cells, including macrophages. The binding between ACh and its receptors will initiate the anti-inflammatory response via the release of anti-inflammatory cytokines such as IL-10 through the inhibition of the nuclear translocation of nuclear factor-кB and the activation of Janus-activated kinase2-signal transducer and activator of transcription factor3. ${ }^{31-34}$ The stimulation of the vagus nerve results in anti-inflammatory effects via a7nAChR-expressing splenic macrophages. ${ }^{35}$ These findings highlight a key role of the PSNS in modulating inflammatory signaling.

Corneal macrophages make up approximately $50 \%$ of the resident corneal leukocytes and primarily populate the central and peripheral corneal stroma. ${ }^{36,37}$ Our recent investigation indicated that corneal macrophages are critical for corneal wound repair and inflammation. ${ }^{9}$ However, these macrophages are heterogeneous, differing with respect to their expression of CC chemokine receptor 2 (CCR2). ${ }^{9}$ CCR2 $^{+}$macrophages are presumably fetal liver- or bone marrow-derived and are proinflammatory. CCR2 ${ }^{+}$ macrophages initiate inflammation in the same way as the classically activated (M1) macrophage subset in the early stage of corneal injury. In contrast, CCR2 ${ }^{-}$macrophages possibly originate from the yolk sac or fetal liver and show an anti-inflammatory phenotype like the alternatively activated (M2) subset during the later stage of wound healing. Moreover, a deficiency in either population results in impaired corneal wound healing through either the promotion or inhibition of inflammatory reactions. ${ }^{9}$

While the regulatory effects of the ANS and its dynamic crosstalk with macrophages on systemic and local inflammation have been well studied, their complex role in orchestrating corneal wound repair remains less clear. To address this issue, we combined a murine model of corneal abrasion with pharmacological tools, a restraint-stress protocol, and cell phenotypic and transcriptional analyses for corneal macrophages to determine the influence of the ANS on the murine corneal wound-healing process. Our results reveal a novel mechanism by which interactions between ANS terminals and corneal tissues trigger or dampen inflammation after corneal abrasion by preferentially expressing different ARs or AChRs, respectively. This study also demonstrates the pharmacological facilitation of corneal reepithelialization and should, therefore, have significant clinical implications.

\section{RESULTS}

The autonomic neural network innervates the mouse cornea To visualize the sympathetic innervation of the mouse cornea, we employed a whole-mount technique with immunofluorescent staining using anti-tyrosine hydroxylase (anti-TH) or antivesicular monoamine transporter 2 (anti-VMAT2) antibodies as the markers of sympathetic nerve fibers. Similarly to other authors, ${ }^{16,17}$ we found that sympathetic nerve fibers are present in normal mouse corneas. However, we detected TH-positive nerve fibers in the corneal epithelium around Regions 2 and 3 (shown in Fig. S-2), some of which spanned the entire lining (Fig. 1a). Most sympathetic fibers in the stroma were located in the subbasal plexus and exhibited positive costaining for $\beta$ tubulin III (Fig. 1b) over the whole cornea. We also visualized fibers in the limbus, primarily running parallel to the blood vessels (Fig. 1c). These results are in line with observations confirmed by Ivanusic et al., ${ }^{19}$ who found that sympathetic nerve fibers exist not only in the mouse corneal stroma but also in the mouse corneal epithelium. Next, to determine the location and distribution of the parasympathetic fibers in the cornea, we used anticholine acetyltransferase (ChAT) antibodies as labels in the corneal limbus and stroma (Fig. 1d). Labeling for ChAT allowed us to conclude that most of the parasympathetic fibers were distributed in the limbus, where they were closely associated with limbal vessels (Fig. 1d). These results suggest that the mouse cornea is under dual control of the sympathetic and parasympathetic systems. 

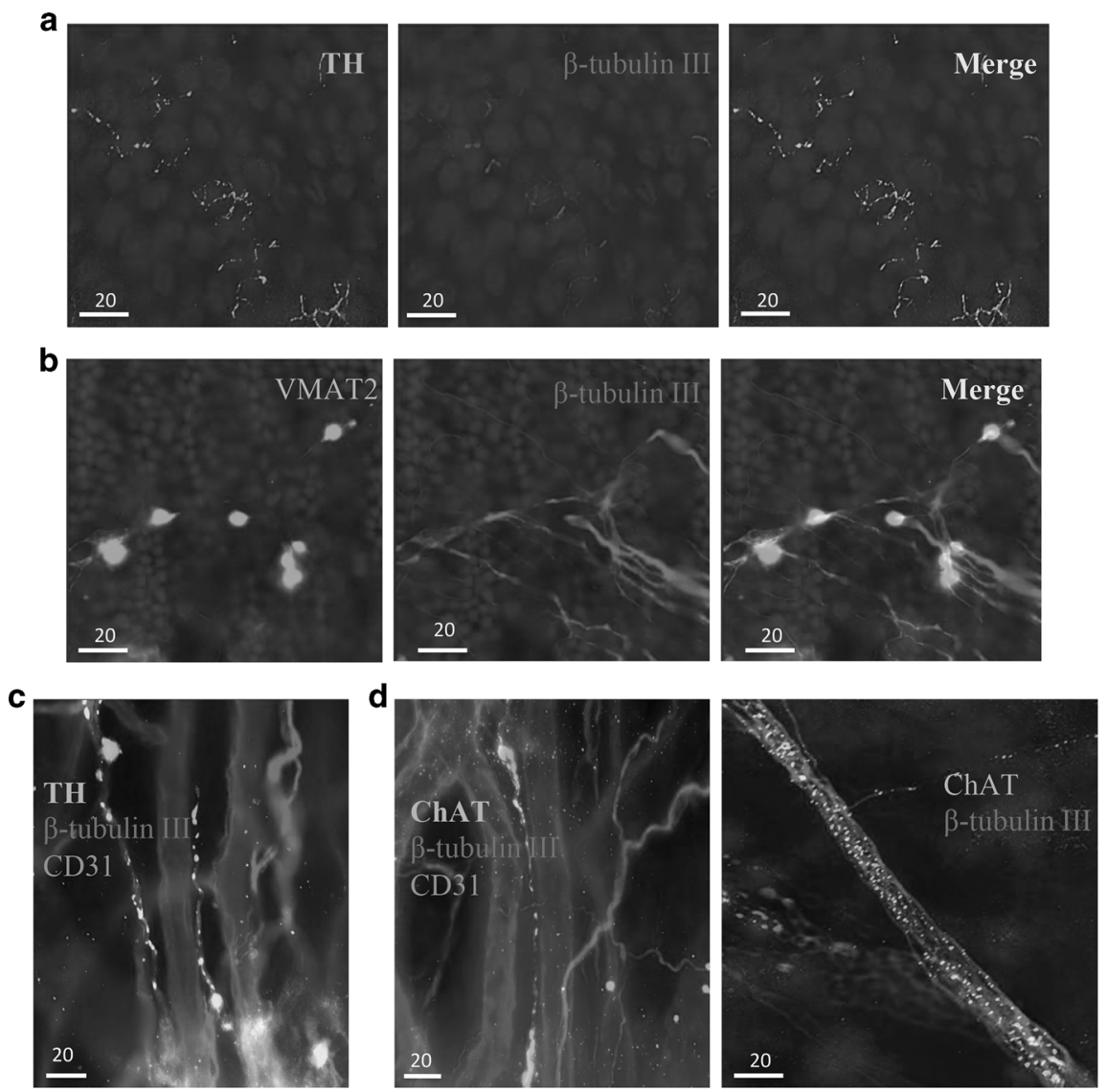

Fig. 1 Autonomic nerve fiber distribution in the mouse cornea. a Sympathetic nerve fibers in the corneal epithelium. Sympathetic fibers (tyrosine hydroxylase [TH]) are shown in green, and corneal nerves ( $\beta$-tubulin III) are shown in red. b Sympathetic nerve fibers distributed in the subepithelial stroma. Sympathetic nerve fibers (vesicular monoamine transporter 2 [VMAT2]) are green, and corneal nerve fibers ( $\beta$-tubulin III) are red. c Sympathetic nerves in the limbus. TH, $\beta$-tubulin III, and CD31 were used to separately stain sympathetic nerve fibers (green), corneal nerve fibers (red), and vessels (blue), respectively. d Parasympathetic nerve fibers (choline acetyltransferase [ChAT]; green) were found in the limbus (left) and stroma (right). Corneal nerve fibers ( $\beta$-tubulin III, red) and vessels (CD31, blue) are also shown. All scale bars $=20 \mu \mathrm{m}$

Sympathetic innervation modulates abrasion-induced reepithelialization and epithelial division

SNS innervation has an important role in the regeneration of organs and tissues, such as the skin, heart, and others. ${ }^{21,38,39}$ Sympathetic denervation accelerates or postpones wound-healing processes depending on the tissue type. Similarly, different kinds of stresses, such as traumatic injury or acute restraint treatment (RST) of animals, trigger the overactivation of the SNS and a massive release of catecholamine into the peripheral circulation to influence healing. ${ }^{40-44}$ To determine the activation status of the SNS after corneal abrasion, we measured the plasma catecholamine concentrations in injured animals at $6,12,18$, and $24 \mathrm{~h}$ after wounding. Abrasion-sham animals that received only intraperitoneal injections of pentobarbital without corneal abrasion were used as a control group. We used the RST model as a stress-related SNS overactivation model to understand the special role of higher SNS activation in corneal wound healing. The results showed that the plasma epinephrine concentration began to rise $6 \mathrm{~h}$ after corneal abrasion (a simple mechanical injury), reached its peak after $24 \mathrm{~h}$, and was significantly higher than in the abrasion-sham control animals (Figure S1). As expected, the RST treatment further increased the plasma epinephrine concentration, indicating enhanced activated sympathetic activity in normal wounded animals.

Corneal epithelial migration and proliferation after abrasion are key processes for rapid reepithelialization. To characterize the role of SNS activation in abrasion-induced corneal reepithelialization and epithelial mitosis, we first induced corneal abrasion in sham control animals and RST animals. We found that the reepithelialization after corneal abrasion in RST animals was significantly slower and the wound did not completely close until $48 \mathrm{~h}$ after abrasion compared with the normal control group (30 h; Fig. 2a, b, left; two-tailed $t$-test, $P<0.01)$. In addition, corneal epithelial division after abrasion in RST animals further decreased compared with the sham control group (Fig. 2b, middle and right; factorialdesign analysis of variance (ANOVA), $P<0.01$ ). This finding indicates that the biased sympathetic activation condition further impaired wound healing after corneal abrasion.

To further demonstrate the role of sympathetic innervation and sympathetic activation in corneal reepithelialization and cell division, we designed systemic and local sympathetic removal models with 6-hydroxydopamine (6-OHDA) and superior cervical ganglionectomy (SCGx), respectively. In the two sham control groups-with only phosphate-buffered saline (PBS) injections or only-neck-skin incisions, respectively-the wounds were typically closed by $30 \mathrm{~h}$ with complete reepithelialization (Fig. 2a). However, we found that, at 12, 18, and $24 \mathrm{~h}$ after abrasion, the percentages of unhealed area were significantly smaller in the 6-OHDA and SCGx groups than in the control animals (Fig. 2a, C, left; factorial-design ANOVA, $P<0.01$ ). Using the same treatment groups, we next investigated how the SNS affects the division of epithelial cells after corneal abrasion. We quantified the number of cells undergoing mitosis at several time points after abrasion and found that cell division peaked $24 \mathrm{~h}$ after abrasion in 
the control group (Fig. 2c, middle). In contrast, cell division peaked at $18 \mathrm{~h}$ after abrasion in both the 6-OHDA-treated and SCGx groups (Fig. 2c, middle). In addition, the increased cell proliferation in injured corneas at $18 \mathrm{~h}$ after corneal abrasion in SCGx-treated mice relative to the controls was confirmed by flow cytometric analyses of cells harvested from the injured corneas (Fig. 2d, left and middle). The number of $\mathrm{Ki}^{+} 7^{+}$corneal cells in the SCGx mice was higher than in the control mice (Fig. 2d, right; factorial-design

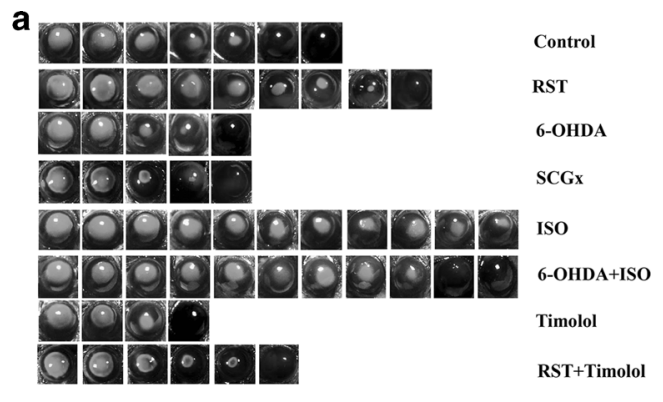

Oh 6 h 12 h 18 h 24 h 30 h 36 h 42 h 48 h 54 h 60 h

Hours after wounding

b
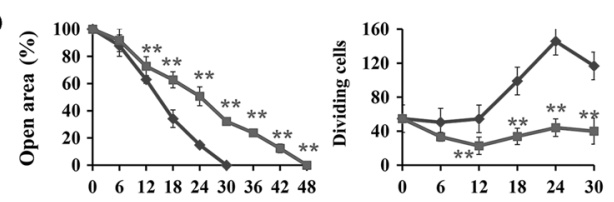

Hours after wounding

C

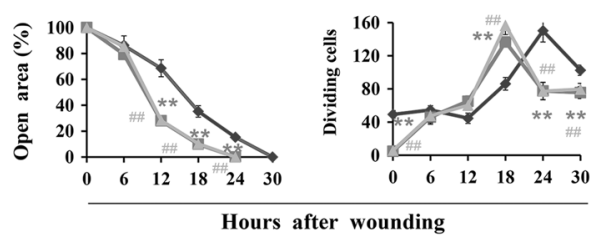

d
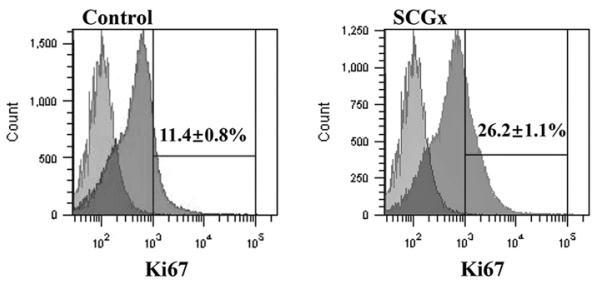

$18 \mathrm{~h}$ after wounding

e
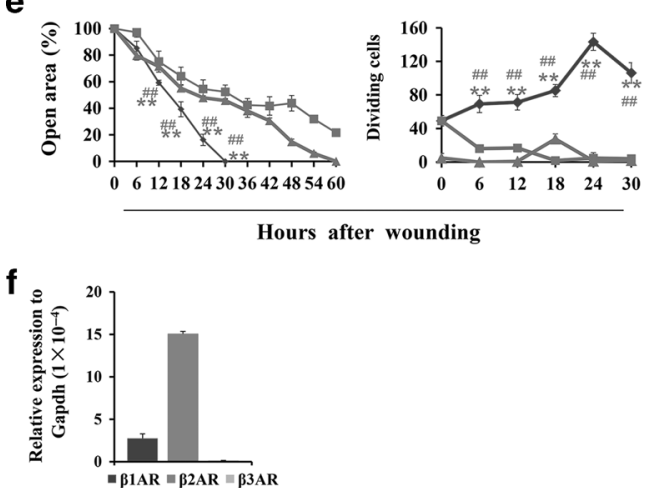

9

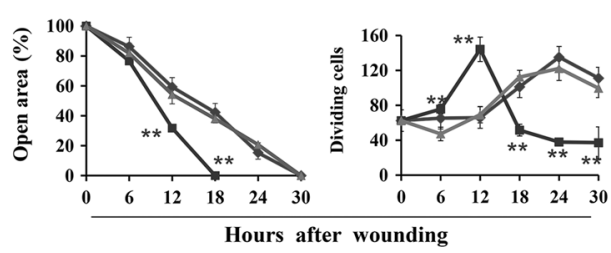

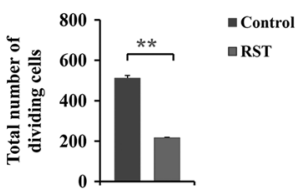
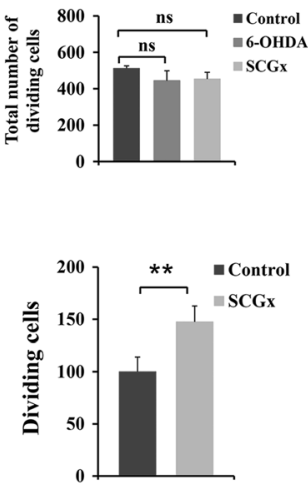
Fig. 2 Effects of sympathetic function on corneal reepithelialization and division. a Fluorescein sodium (green) was used to visualize the wounded areas in control mice and those treated with timolol, SCGx, 6-OHDA, 6-OHDA+ISO, ISO, RST, or RST+timolol ( $n=6$ mice at each time point). b, c, e, and $\mathbf{g}$ (left) The corneal wound repair rates were faster than the control rates in the 6-OHDA (c), SCGx (c), and timolol (g) groups; slower in the RST (b), ISO (e), and 6-OHDA+ISO (e) groups; and did not change in the RST+timolol (g) group ( $n=6$ mice at each time point. b, $\mathbf{c}, \mathbf{e}$, and $\mathbf{g}$ (middle) Numbers of dividing cells at each time point after corneal abrasion ( $n=6$ corneas at each point). b, $\mathbf{c}, \mathbf{e}, \mathbf{g}$ (right) Total number of dividing cells from 0 to $30 \mathrm{~h}$ after corneal wounding. Although the total numbers for the timolol (g, right), 6-OHDA, and SCGx groups (c, right) did not differ from the controls, those for the RST (b, right), ISO (e, right), and 6-OHDA+ISO (e, right) groups were significantly lower. No difference was observed in the RST+timolol group $(\mathbf{g})\left(n=6\right.$ corneas at each point). $\mathbf{d}$ (left and middle) Ki67 ${ }^{+}$cells were phenotyped and analyzed by flow cytometry $18 \mathrm{~h}$ after corneal wounding in the SCGx and control groups. (d, right) $18 \mathrm{~h}$ after corneal wounding, more $\mathrm{Ki} 67^{+}$cells were found in the SCGx group than in the control group ( $n=3$ independent experiments, 10 mice per experiment). $f$ In the mouse cornea, $\beta 2 A R$ is the predominate $\beta A R$. Dividing cells were counted in 9 regions (Regions $1,2,3,4,5,4{ }^{\prime}, 3^{\prime}, 2^{\prime}$, and 1' in Figure S2) from limbus to limbus ( $n=6$ corneas at each point). The asterisk symbols indicate comparisons between different the experimental groups and the control. ${ }^{*} P<0.01,{ }^{\# \#} P<0.01, \mathrm{~ns}=$ nonsignificant. RST restraint stress model, 6-OHDA chemical sympathectomy, SCGx superior cervical ganglionectomy, ISO isoproterenol, a $\beta A R$ agonist, 6-OHDA+ISO topical treatment with ISO after chemical sympathectomy, timolol a $\beta A R$ antagonist, RST+timolol topical treatment with timolol after RST

ANOVA, $P<0.01)$. These data suggest that local innervation and activation of the SNS after abrasion inhibit reepithelialization and cell division of the cornea.

Many effects of the SNS on tissues and organs are mediated by the release of epinephrine and norepinephrine. To confirm the impaired effect of the SNS innervation and activation on the corneal wound healing described above, we tested the topical effects of isoproterenol (ISO), an isopropyl analog of epinephrine, as a nonselective $\beta A R$ agonist to locally reconstitute epinephrine in both the 6-OHDA-treated and the balanced salt solution (BSS)sham control groups. As expected, topical ISO administration in the BSS-sham control animals delayed the wound-healing process until at least $60 \mathrm{~h}$ after abrasion; this also occurred in the 6-OHDAtreated animals (Fig. 2e, left; factorial-design ANOVA, $P<0.01$ ). Additionally, epithelial cell division in both groups was inhibited to a large degree after abrasion (Fig. 2e, middle and right; factorialdesign ANOVA, $P<0.01$ ). These data suggest that the woundhealing delay caused by SNS innervation and activation is mainly mediated by the CChs released after abrasion.

The effects of stress hormones such as norepinephrine and epinephrine resulting from sympathetic activation are mediated through binding to $\beta 1-, 2-$, and 3-ARs on target cells. To select appropriate AR agonists and antagonists, we used reverse transcription quantitative-polymerase chain reaction (qRT-PCR) to obtain the expression profiles of genes encoding subunits of the AR types for neurotransmitters released by the SNS in the mouse cornea. The results showed that, among the $\beta A R s, \beta 2-$ gene expression dominated over $\beta 1$-gene expression in the mouse cornea, but little $\beta 3$ expression was found (Fig. 2f). Thus, in the next step, we topically applied the nonselective $\beta A R$ blocker timolol to observe the effect of $\beta A R$ blockage on reepithelialization and cell division. We found that, at 12,18 , and $24 \mathrm{~h}$ after abrasion, the percentages of unhealed area were significantly smaller in the topical timolol groups than in the controls (Fig. 2a, $\mathrm{g}$, left; factorial-design ANOVA, $P<0.01$ ). Using the same treatment groups, we next investigated the effect of topical timolol on the division of epithelial cells after corneal abrasion. We quantified the number of cells undergoing mitosis at several time points after abrasion and found that cell division peaked $24 \mathrm{~h}$ after abrasion in the control group. In contrast, cell division peaked at $12 \mathrm{~h}$ after abrasion in the timolol groups (Fig. $2 \mathrm{~g}$, middle). To further confirm the therapeutic effect of the $\beta A R$ antagonist on the impaired wound healing of the cornea in biased SNS activation animals, we also observed the course of reepithelialization and epithelial division in RST+topical timolol animals. As expected, the reepithelialization and the number of dividing cells in the RST+timolol group almost reached normal levels compared to both the RST and the normal control groups (Fig. $2 \mathrm{~g}$, right). These results therefore indicate that the biased activation of the SNS inhibits both reepithelialization and epithelial proliferation after corneal abrasion and that blocking the $\beta A R$ signaling pathway reverses this state of inhibition. Altogether, these data indicate that the blockage of the $\beta A R$ signaling pathway was beneficial for the faster wound closure in both normal and highly biased sympathetic tones.

Sympathetic innervation stimulates abrasion-induced neutrophil recruitment to the wound

The SNS influences the immune system and therefore controls the onset of inflammation at the cellular and molecular levels. ${ }^{45}$ To investigate how this SNS innervation and activation affects neutrophil recruitment to the wounded cornea, we compared the dynamics of neutrophil influx to the wound area in three different regions (the limbus, wound margin, and wound center of the corneal stroma; Figure S2) among the eight groups of mice laid out in the last section: normal control, RST as a stress-related SNS overactivation model, 6-OHDA and SCGx as SNS removal models, topical ISO administration, 6-OHDA-pretreated plus topical ISO administration as a reconstitution model, epinephrine, topical timolol, and RST+topical timolol as a topical $\beta A R$ antagonism model. The neutrophil influx to the wound was quantified by the number of $\mathrm{Ly}_{6 \mathrm{G}}{ }^{+}$neutrophils in the stroma of the limbus, the wound margin, and the wound center every $6 \mathrm{~h}$ for $30 \mathrm{~h}$ after abrasion (Fig. 3). In each control run, the number of neutrophils in each region peaked $18 \mathrm{~h}$ after corneal abrasion (Fig. 3).

As expected, we found that RST mice showed an in-advance peak of leukocyte trafficking in the limbus (Fig. 3a). The total number of neutrophils from 0 to $30 \mathrm{~h}$ after abrasion significantly increased compared to the control group (Fig. 3a; factorial-design ANOVA, $P<0.01)$. However, there was little effect on the neutrophil accumulation at the wound margin and center (Fig. 3a).

The number of neutrophils in the SCGx and 6-OHDA-treated groups peaked at a similar time as the control group, but the actual peak values were significantly lower in the SCGx- and 6OHDA-treated groups than in the control group (Fig. 3b; factorialdesign ANOVA, $P<0.01$ ). This result in the SCGX group was confirmed by a flow cytometric analysis of cells harvested $18 \mathrm{~h}$ after corneal abrasion (Fig. 3f, left and middle); the number of $\mathrm{Ly}_{6 \mathrm{G}}{ }^{+}$neutrophils in the wounds of SCGx-treated mice was lower than in the control mice (Fig. 3f, right; factorial-design ANOVA, $P<0.01$ ). Together, these data suggest that the removal of both systemic and local SNS innervation suppresses the influx of neutrophils to the wound.

To confirm whether neutrophil recruitment to the wounded cornea is mediated by the release of epinephrine and norepinephrine from the SNS, we checked the topical effects of ISO as a nonselective $\beta A R$ agonist in locally reconstituting epinephrine in both the 6-OHDA-treated and PBS-sham control groups. As expected, the neutrophil influx into three different areas peaked at $12 \mathrm{~h}$ in the animals with topical ISO administered; in the PBSsham control animals, this was shifted $6 \mathrm{~h}$ earlier (Fig. 3c, three 


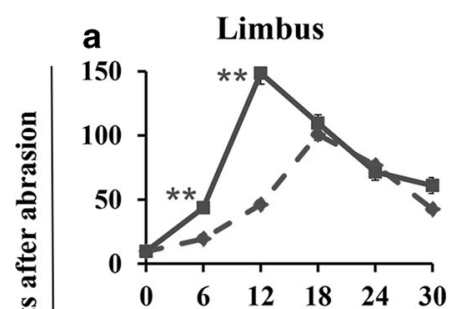

b

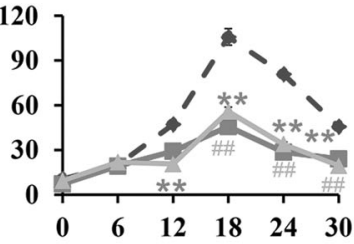

C

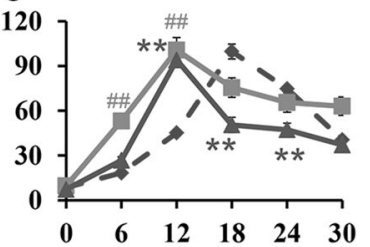

d

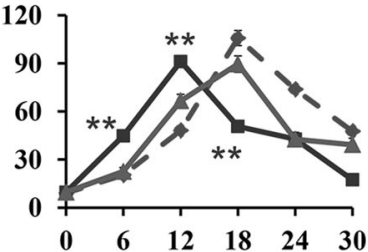

e

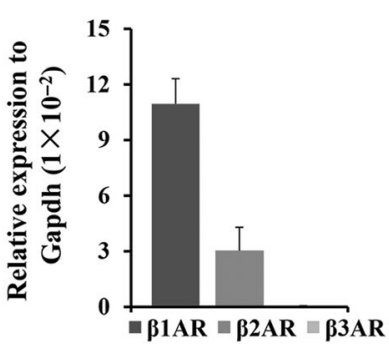

Wound margin

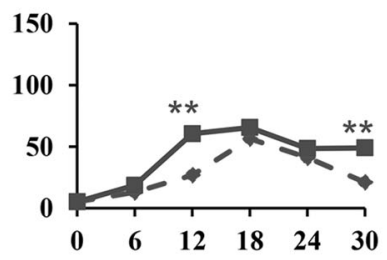

Wound center
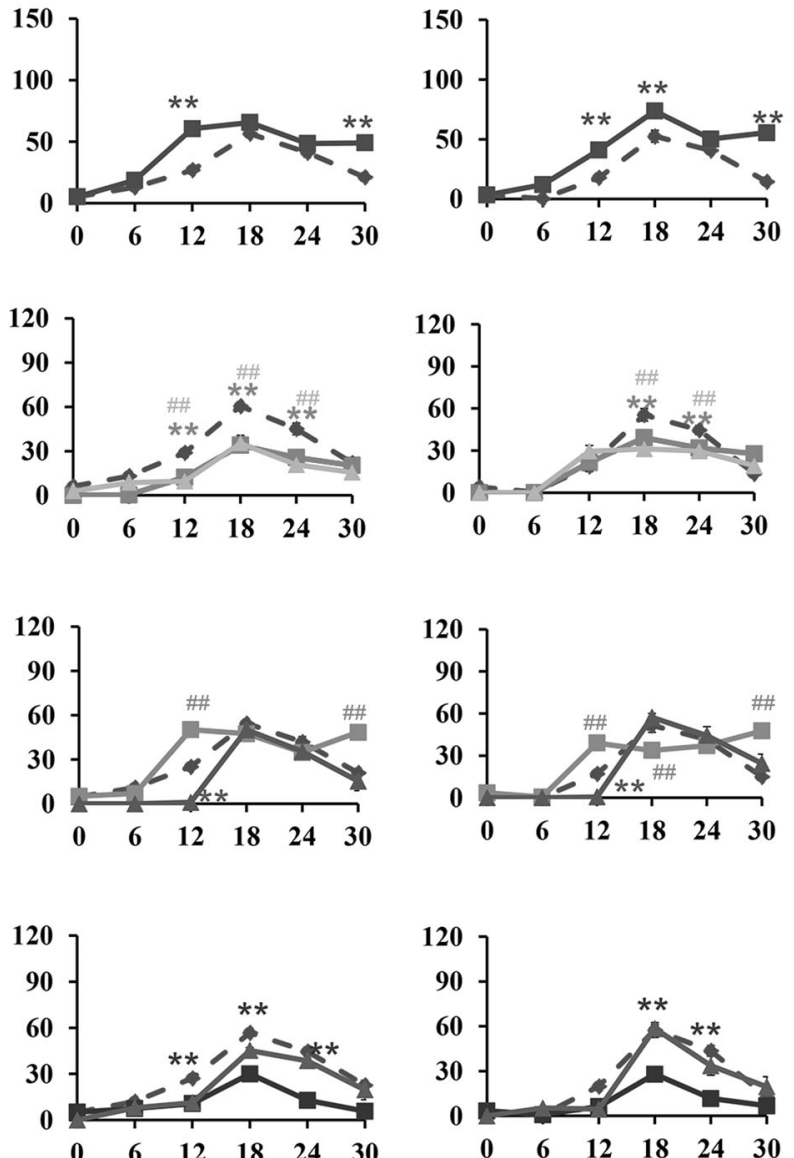

f

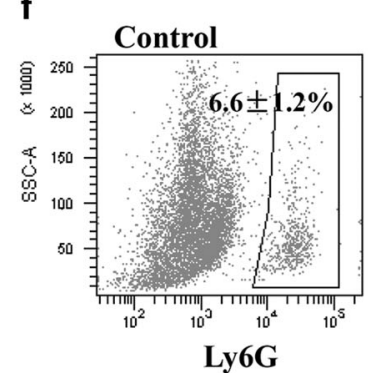

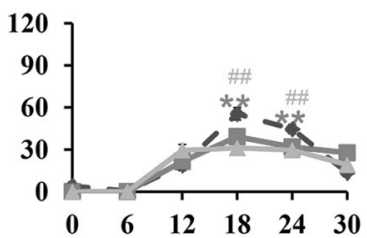
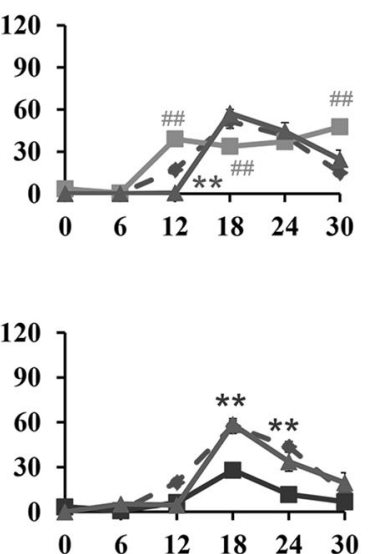

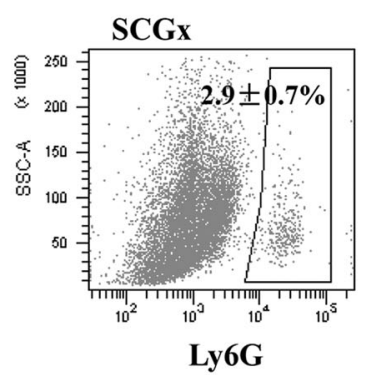

Total number

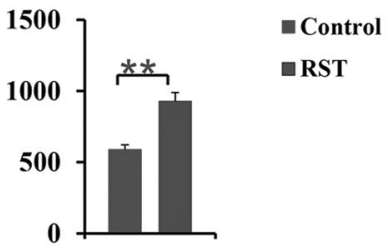

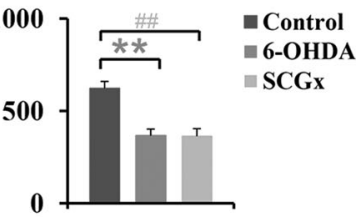
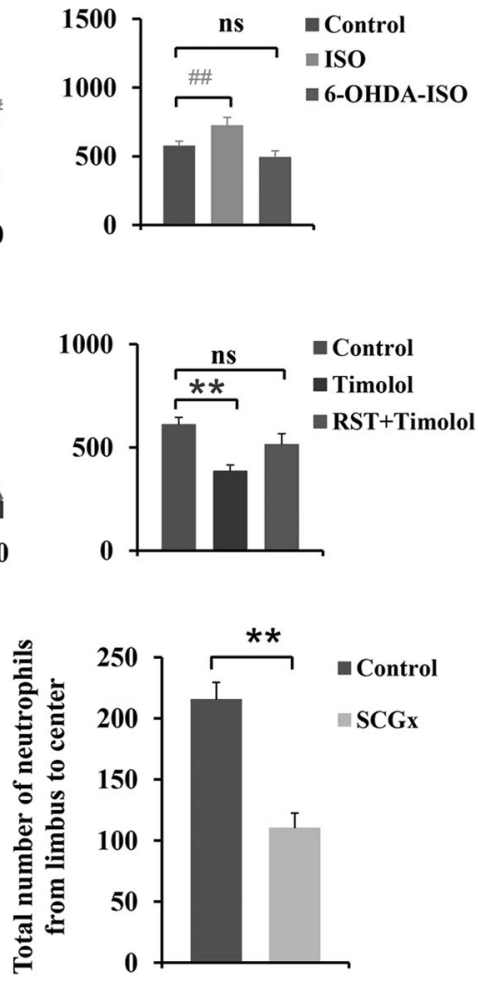

$18 \mathrm{~h}$ after wounding

Fig. 3 Influence of sympathetic function on neutrophil trafficking to injured corneas. a-d Neutrophils trafficking to the limbus, wound margin, and wound center of the corneal stroma from 0 to $30 \mathrm{~h}$ after abrasion were counted. Enhancing the sympathetic activity with RST (a) and topical ISO (c) raised the number of neutrophils while blocking sympathetic activity with 6-OHDA (b), SCGx (b), or timolol (d) lowered the number of neutrophils ( $n=6$ corneas at each point). The far-right panels from a and $\mathbf{d}$ show the changes of total neutrophil numbers after surgical and pharmacological manipulations. e The neutrophil population, harvested from wounded corneas at $18 \mathrm{~h}$ after wounding, dominantly expressed $\beta 1 A R$. f Wound neutrophils (Ly6G ${ }^{+}$cells) harvested at $18 \mathrm{~h}$ after wounding from the corneas of either control mice (f, left) and mice treated with SCGx (f, middle) were phenotyped and analyzed by flow cytometry, respectively; the control group contained more neutrophils than the SCGx group (f, right; $n=3$ independent experiments, 10 mice per experiment). The asterisk symbol indicates the comparison between different experimental groups and control group. ${ }^{* *} P<0.01,{ }^{\# \#} P<0.01$. RST restraint stress model, 6-OHDA chemical sympathectomy, SCGx superior cervical ganglionectomy, ISO isoproterenol, a $\beta A R$ agonist, 6-OHDA+ISO topical treatment with ISO after chemical sympathectomy, timolol a $\beta$ AR antagonist, RST+timolol topical treatment with timolol after RST, SSC slide scatter

left-most graphs). The cumulative total number in the topical ISOPBS-sham control animals was significantly higher than in the topical PBS control group (Fig. 3c, far-right figure; factorial-design ANOVA, $P<0.01$ ). However, in topical ISO-6-OHDA-treated animals, the total number of neutrophils was similar to that of the control group (Fig. 3c). These data suggest that the wound-healing delay caused by SNS innervation and overactivation may be mediated mainly by CChs released from the SNS.

The effects of stress hormones such as norepinephrine and epinephrine regulate the immune response and inflammation 

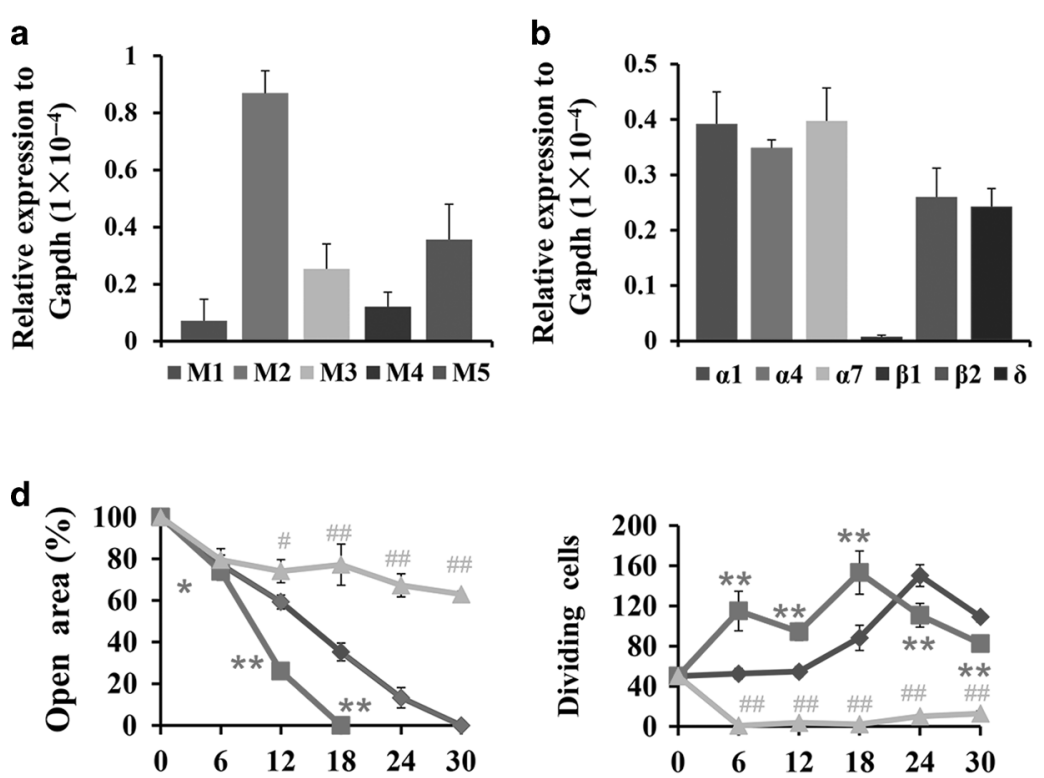

e
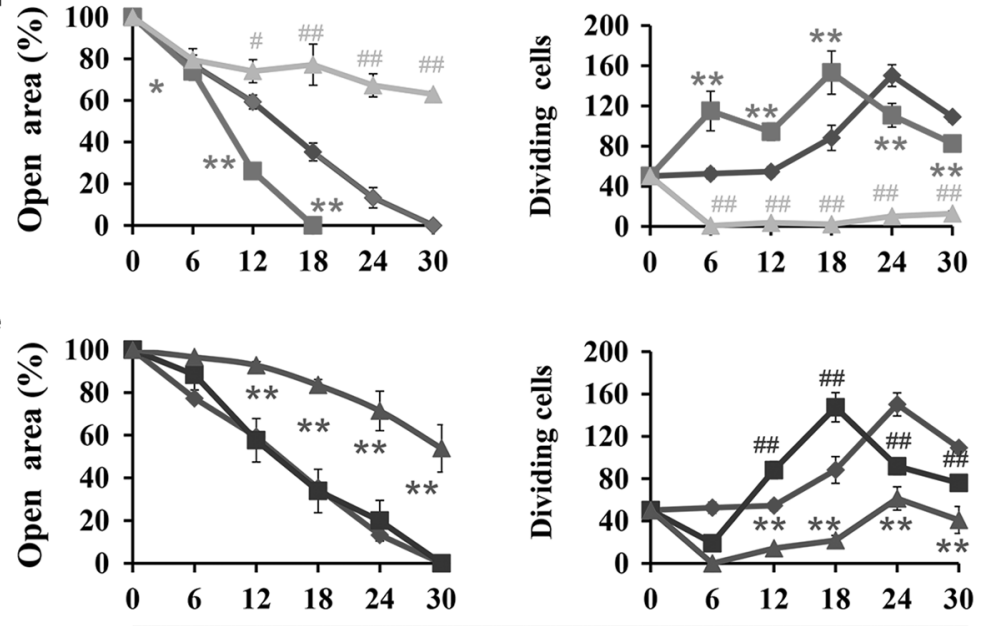

C
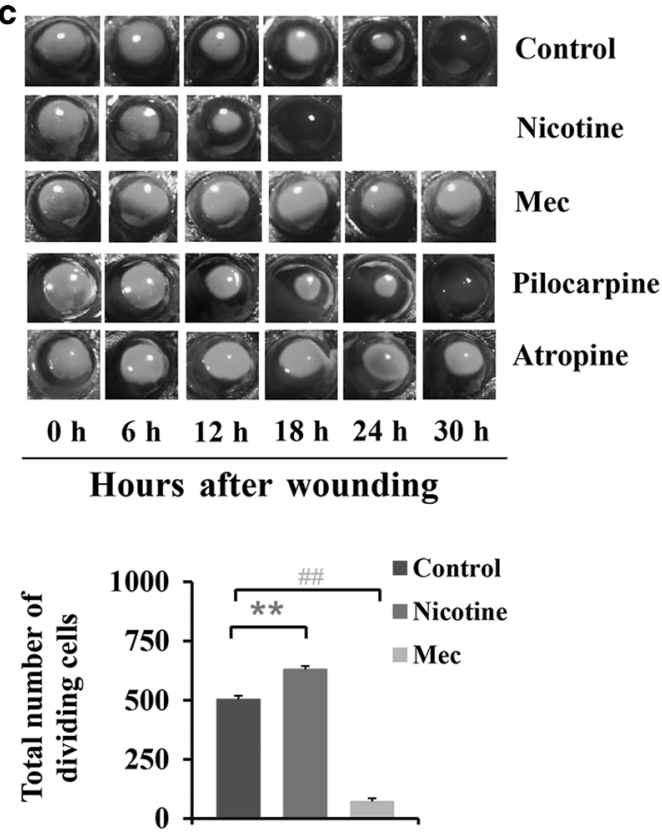

\section{Hours after wounding}

Fig. 4 Effects of parasympathetic function on corneal reepithelialization and division. a In the mouse cornea, M2 is the predominate muscarinic acetylcholine receptor, and $\mathbf{b} \alpha 1,4$, and 7 nicotinic receptors are the predominate nicotinic receptors. $\mathbf{c}$ Fluorescein sodium (green) was applied to visualize the wounded areas in the control mice and those treated with $\alpha 7 n A C h R$ and mAChR agonists (nicotine and pilocarpine, respectively) or antagonists (mecamylamine and atropine, respectively; $n=6$ mice at each point). $\mathbf{d}$, e (left) Corneal wound repair rates following treatment with nicotine, mecamylamine (d), and atropine, but not pilocarpine (e), were markedly different from those in the control group ( $n=6$ mice at each point). d, e (middle) The numbers of dividing cells at each time point after corneal abrasion ( $n=6$ corneas at each point). d, e (right) Total number of dividing cells from 0 to $30 \mathrm{~h}$ after corneal wounding. The total numbers were increased in the nicotine group (d) and decreased in the mecamylamine (d) and atropine (e) groups compared to the controls. No change was observed in the pilocarpine group (c; $n=6$ corneas at each point). The asterisk symbols indicate comparisons between different experimental groups and the control. ${ }^{*} P<0.05,{ }^{* *} P<0.01,{ }^{\#} P<0.05,{ }^{\# \#} P<0.01$. Nicotine an $\mathrm{nAChR}$ agonist, Mec mecamylamine, an nAChR antagonist, pilocarpine an mAChR agonist, atropine an $\mathrm{mAChR}$ antagonist, $\alpha 7 \mathrm{nAChR} \alpha-7$ nicotinic acetylcholine receptor, $\mathrm{mAChR}$ muscarinic ACh receptor

through the binding of different ARs expressed on the immune cells. ${ }^{46}$ To understand the AR types expressed on neutrophils, we sorted the neutrophil population from the wounded corneas $18 \mathrm{~h}$ after abrasion. The transcriptional genes encoding subunits of the AR types examined via the qRT-PCR technique showed that these neutrophil populations dominantly expressed $\beta 1 A R$ (Fig. 3e). To observe the effect of a $\beta A R$ blockade on neutrophil recruitment to the wounded cornea, we topically administered timolol after abrasion. We quantified the number of recruited neutrophils in the different regions of the wounded corneas at several time points after abrasion and found that the number of neutrophils in the limbus peaked $12 \mathrm{~h}$ after abrasion and shifted $6 \mathrm{~h}$ earlier than that of the control and RST+timolol-treated groups but not in the wound margin and wound center (Fig. 3d). Compared to the normal control group, the number of neutrophils at both the wound margin and center were significantly lower (Fig. 3d; factorial-design ANOVA, $P<0.01$ ). However, the cumulative total number from 0 to $30 \mathrm{~h}$ in the timolol-treated groups was lower than in the normal control group (Fig. 3d, far-right figure; factorial-design ANOVA, $P<0.01$ ). To determine the effects of a $\beta A R$ blockade on excessive neutrophil recruitment due to the SNS overreaction in the RST model, we quantified the number of recruited neutrophils in the different regions of the wounded corneas at several time points after abrasion. We found that the number of neutrophils in three different areas and the cumulative total number from 0 to $30 \mathrm{~h}$ after abrasion were restored to the level seen in normal control animals (Fig. 3d). Altogether, these data indicate that a blockage of the $\beta A R$ signaling pathway suppresses neutrophil recruitment to the wounded cornea and could alleviate excessive inflammation owing to an overreaction of the SNS.

Cholinergic parasympathetic signaling regulates wound closure The presence of parasympathetic fibers in the corneal limbus and corneal stroma led us to evaluate the role of the PSNS in corneal epithelial wound repair. Postganglionic PSNS neurons activate classic effector organs mainly by releasing $A C h$ onto mAChRs (M1-M5) and nAChRs (containing a1-10, $\beta 1-4$, and $Y$ units) 
expressed on nonclassical target cells, including corneal cells. ${ }^{47}$ To select appropriate agonists and antagonists to mAChRs and nAChRs to work on murine corneal cells, we detected the transcriptional expression profile of acetylcholine receptors ( $m A C h R s$ and nAChRs) in the normal murine cornea with the qPCR technique. The results showed that, among the mAChRs (M1-5), M2 and M5 were expressed more than the others (Fig. 4a). Of the six main $\mathrm{N}$-receptor subunits ( $\alpha 1, \alpha 4, \alpha 7, \beta 1, \beta 2$, and $\delta), \alpha 1$, $a 4$, and $a 7$ were the ones predominately expressed in the cornea (Fig. 4b). Thus corneal cells in mice predominately express the $M$ $(2,5)$ AChRs and the $a(1,4,7)$ nAChRs.

To confirm the effects of the nAChR signaling pathway on reepithelialization and cell division after abrasion, we applied nicotine topically as an agonist for a variety of pentameric nAChRs and mecamylamine as a noncompetitive nicotinic receptor antagonist after inducing corneal wounds. The results showed that reepithelialization in the control group was completed $30 \mathrm{~h}$ after corneal abrasion, as before (Fig. 4c). In contrast, wound closure was completed $18 \mathrm{~h}$ after abrasion in the nicotine-treated group (Fig. 4c). Interestingly, wound closure was not completed until $48 \mathrm{~h}$ after abrasion in the mecamylamine-treated group (Fig. 4c). In addition, we counted and compared the differences in the number of corneal epithelial cells among the topical nicotinetreated, the topical mecamylamine-treated, and the control groups. The results showed that the numbers of dividing cells in the nicotine-treated group significantly increased at 6, 12, and $18 \mathrm{~h}$ after abrasion (Fig. 4d, middle; factorial-design ANOVA,
$P<0.01)$. The cumulative total number of dividing cells from 0 to $30 \mathrm{~h}$ after abrasion was also significantly increased compared to the control group (Fig. 4d, right; factorial-design ANOVA, $P<0.01$ ). However, compared to the control group, the number of dividing cells in the mecamylamine-treated group was significantly decreased at 12, 18, 24, and $30 \mathrm{~h}$ after abrasion (Fig. 4d, middle; factorial-design ANOVA, $P<0.01$ ). In contrast to the nicotinetreated group, the cumulative total number of dividing cells in the mecamylamine-treated group from 0 to $30 \mathrm{~h}$ after abrasion was significantly decreased compared to the control group (Fig. 4d, right; factorial-design ANOVA, $P<0.01$ ). These data suggest that the engagement of $n A C h R s$ alters reepithelialization. The pharmacological stimulation of $\mathrm{nAChRs}$ accelerates postabrasion corneal reepithelialization and increases the number of dividing cells.

In the next step, we topically applied atropine as a specific antagonist against muscarinic receptors and pilocarpine as a nonselective muscarinic receptor agonist to observe the role of mAChRs in reepithelialization after corneal abrasion. The results showed that atropine significantly inhibited corneal reepithelialization compared to the control mice (Fig. 4e; two-tailed $t$-test, $P<0.01)$, with the wound area remaining open until at least $30 \mathrm{~h}$ after abrasion (Fig. 4e). Unexpectedly, pilocarpine did not have any significant effect on mouse reepithelialization in this study (Fig. 4c, e). We next examined whether muscarinic receptors affect postabrasion cell division by counting mitotic cells in the corneal epithelium from limbus to limbus. In the control group, the mitotic
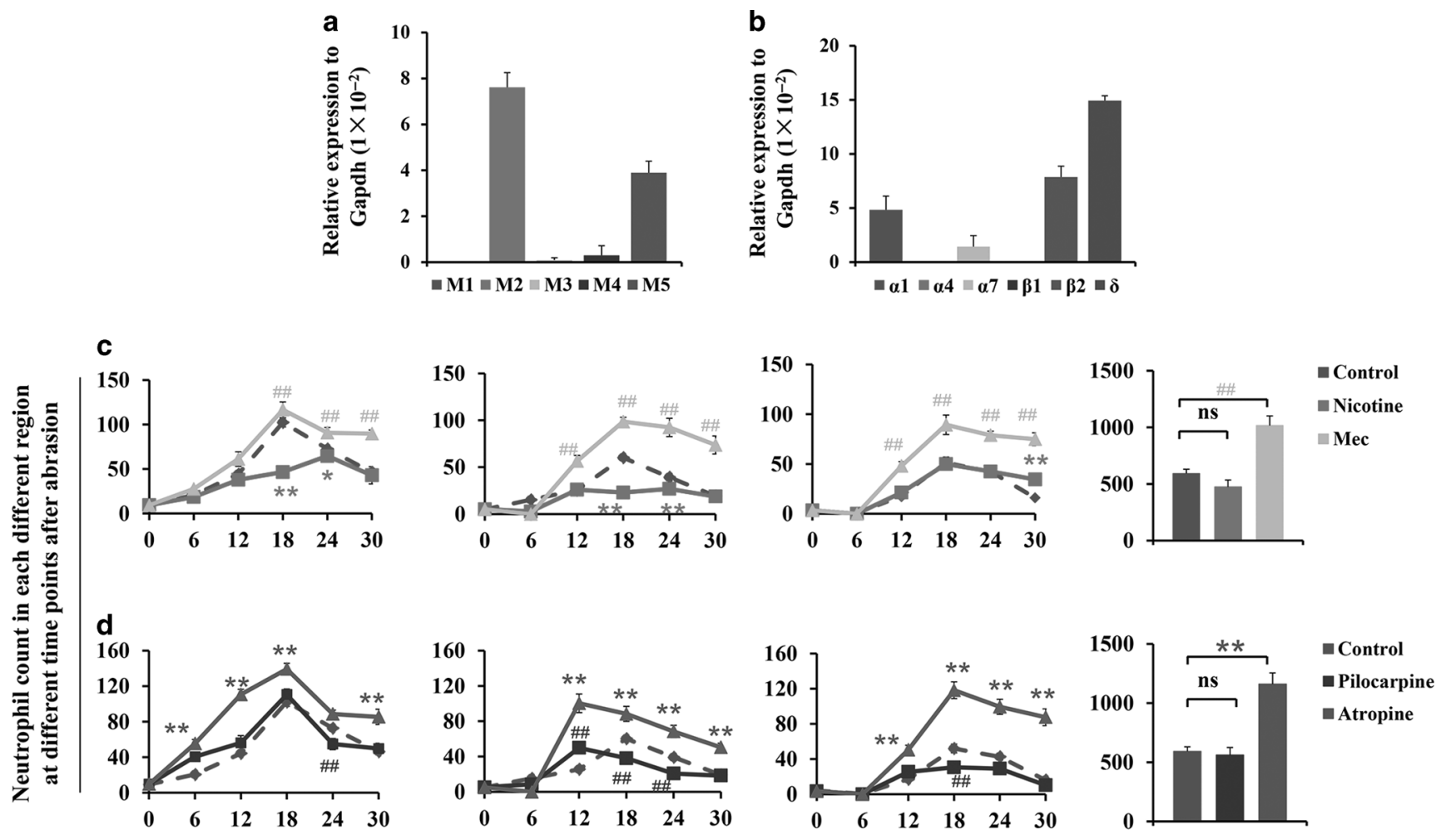

Hours after wounding

Fig. 5 Influence of parasympathetic fibers on neutrophil trafficking to injured corneas. a In the neutrophil population, harvested from wounded corneas at $18 \mathrm{~h}$ after wounding, $\mathrm{M} 2$ is the predominate muscarinic acetylcholine receptor. $\mathbf{b}$ In the neutrophil population, harvested from wounded corneas at $18 \mathrm{~h}$ after wounding, the $\alpha 1,4$, and 7 nicotinic receptors are the predominate nicotinic receptors. $\mathbf{c}$, $\mathbf{d}$ Neutrophils trafficking to the limbus, wound margin, and wound center region of the corneal stroma from 0 to $30 \mathrm{~h}$ after abrasion were numerated in the parasympathetic pharmacological manipulations ( $n=6$ corneas at each point). The asterisk symbols indicate comparisons between different experimental groups and the control. ${ }^{*} P<0.05,{ }^{* *} P<0.01$, and ${ }^{\# \#} P<0.01$ comparing groups as indicated in the figure. Nicotine an nAChR agonist, Mec mecamylamine, an nAChR antagonist, pilocarpine an mAChR agonist, atropine an mAChR antagonist 

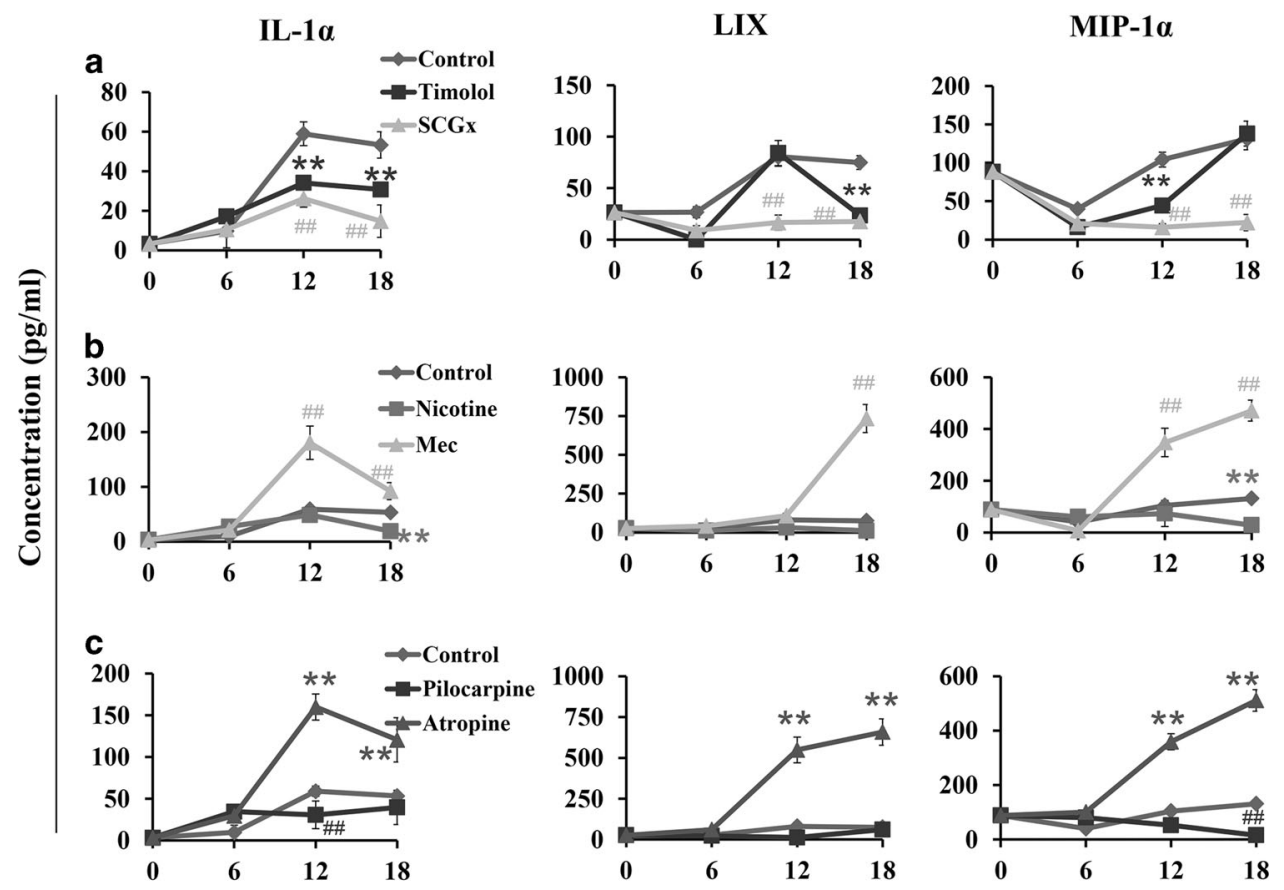

Hours after wounding
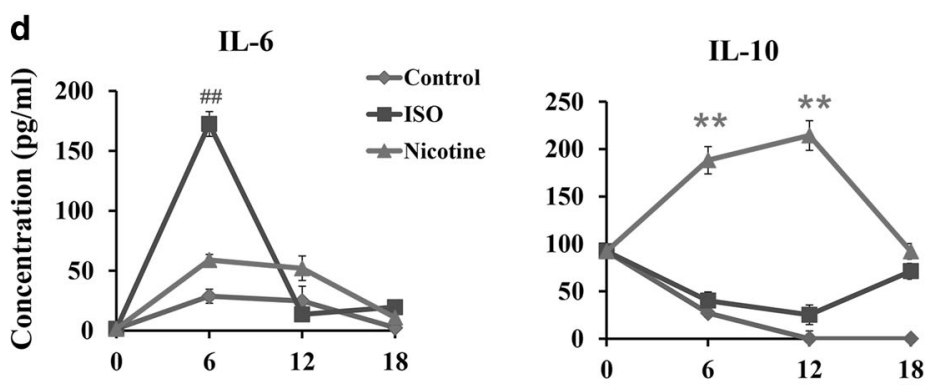

Hours after wounding

Fig. 6 Influence of the autonomic nervous system on local cytokine release after corneal abrasion. Quantification of the cytokines IL-1 $\alpha$, LIX, MIP-1 $\alpha$, IL-6, and IL-10 in the corneal homogenate samples at 0,6, 12, and $18 \mathrm{~h}$ after abrasion was performed using the sandwich ELISA technique to observe the influence of sympathetic (topical timolol and SCGx in a and topical ISO in d) and parasympathetic (topical nicotine and Mec in b, topical pilocarpine and atropine in $\mathbf{c}$, and topical nicotine in d) signaling pathways on the local release of the associated inflammatory molecules. Pooled data were obtained from three different experiments. The data are presented as the mean \pm SD $(n=3$ independent experiments, 5 mice (10 corneas) at each time point). Asterisk symbols indicate comparisons between different experimental groups and the control. ${ }^{* *} P<0.01,{ }^{\#} P<0.01$. Timolol a $\beta A R$ antagonist, ISO isoproterenol, a $\beta A R$ agonist, SCGX superior cervical ganglionectomy, Nicotine an nAChR agonist, Mec mecamylamine, an nAChR antagonist, Pilocarpine an mAChR agonist, Atropine an mAChR antagonist

cells began to increase at $12 \mathrm{~h}$ and peaked at $24 \mathrm{~h}$ (Fig. 4e). In the topical atropine-treated group, the number of epithelial cell divisions significantly decreased at $12,18,24$, and $30 \mathrm{~h}$ after abrasion compared to the control mice (Fig. 4e, middle; factorialdesign ANOVA, $P<0.01)$. Unexpectedly, in the pilocarpine-treated group, the peak of cell division shifted $6 \mathrm{~h}$ earlier than the control group and the atropine-treated group (Fig. 4e, middle). However, the total number of cell divisions from 0 to $30 \mathrm{~h}$ did not change (Fig. 4e, right). These data suggest that the local blockage, not activation, of mAChRs significantly influenced the reepithelialization and epithelial cell division.

Cholinergic parasympathetic signaling regulates abrasion-induced neutrophil recruitment to the wounded cornea

Stimulation of the PSNS is known to inhibit the release of proinflammatory cytokines in infected or injured tissues. ${ }^{48,49}$
Immune cells express some $\mathrm{nAChR}$ subunits (a1-10) to varying degrees and express all mAChRs $(\mathrm{M} 1-\mathrm{M} 5) .{ }^{50}$ However, this expression has not been demonstrated in the cornea, especially with regard to neutrophil infiltration during wound healing. To observe how PSNS activity affects postinjury neutrophil influx, we first sorted and purified $\mathrm{Ly}_{6 \mathrm{G}}{ }^{+}$neutrophils from wounded corneas $18 \mathrm{~h}$ after abrasion and then checked the transcriptional genes encoding subunits of mAChRs and nAChRs. However, our data showed that this neutrophil population dominantly expressed two muscarinic acetylcholine receptors, M2 and M5 (Fig. 5a), and the $a 1, \alpha 7, \beta 2$, and $\delta$ subunit of nAChRs (Fig. $5 b$ ).

In the next step, we compared and analyzed the dynamics of neutrophil trafficking to the wounded corneas of nicotinic receptor agonist nicotine- and antagonist mecamylamine-treated mice. The numbers of neutrophils in the corneal limbus and wound edge area in the nicotine group were significantly lower 

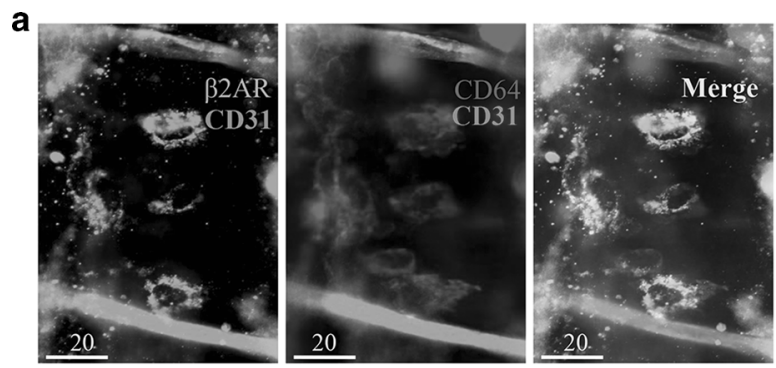

C

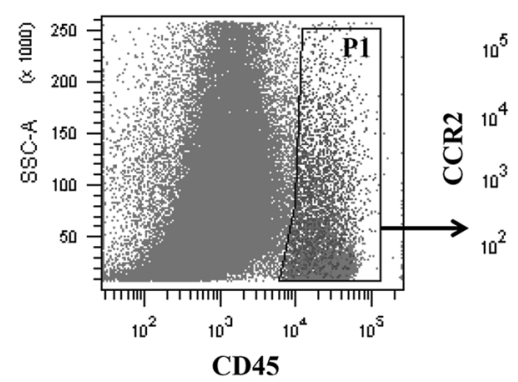

e

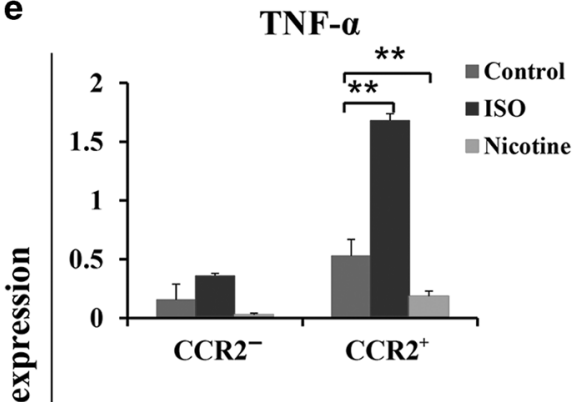

IL-10

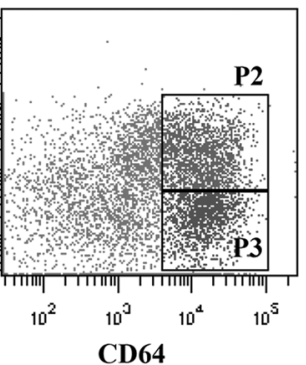

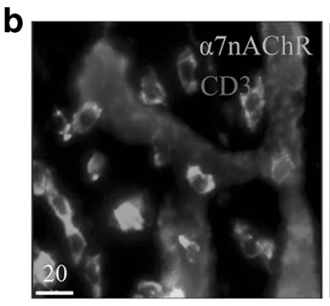
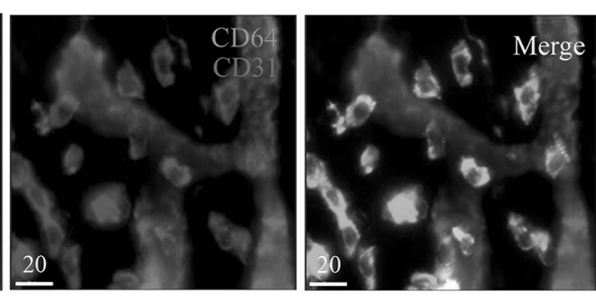

d

B2AR

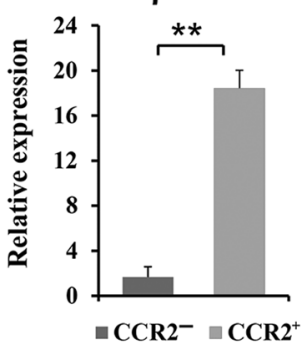

$\alpha 7 n A C h R$

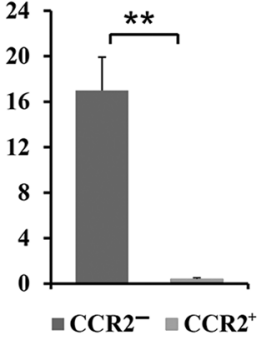

IL-6

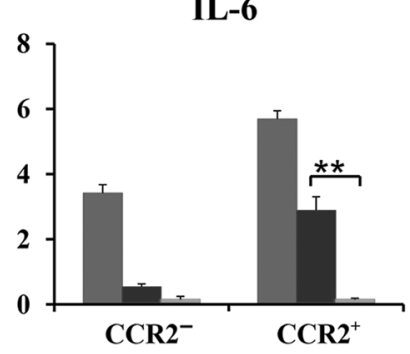

Arg1
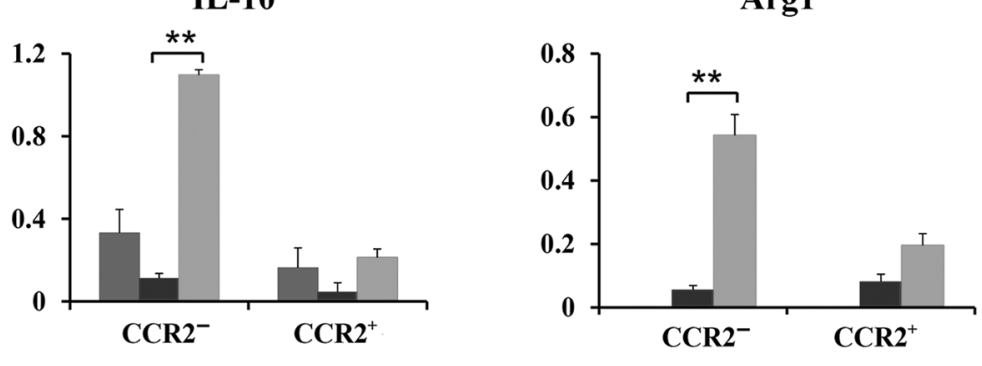

$6 \mathrm{~h}$ after wounding

Fig. 7 The expression of adrenergic, nicotinergic, and muscarinic receptors in corneal macrophages. a Immunofluorescence shows the distribution of $\beta 2 A R$ (green), macrophages (CD64, red), and vascular tissue (CD31, blue) in the corneal limbus. b Macrophages (CD64, blue) also expressed $\alpha 7 n A C h R$ (green). Limbal vessels are marked by CD31 (red). c CD45 ${ }^{+} \mathrm{CD} 64^{+} \mathrm{CCR} 2^{-}$and $\mathrm{CD} 45^{+} \mathrm{CD} 64^{+} \mathrm{CCR} 2^{+}$macrophages (right) were phenotyped and sorted from a presorted $\mathrm{CD}_{4} 5^{+}$cell population (left) of collagenase-digested single-cell suspension in the normal corneas by flow cytometry ( $n=3$ independent experiments, 10 mice per experiment). $\mathbf{d}$ Expression analysis showed that $\beta 2 A R$ and $\alpha 7 n A C h R$ are preferentially expressed in $\mathrm{CCR}^{+}$and CCR2 ${ }^{-}$corneal macrophages, respectively. e CCR2 ${ }^{+}$and $\mathrm{CCR}^{-}$macrophages were sorted $^{-}$ from 6-h corneal wounds that were treated with either topical ISO or nicotine, and the gene expression of the cytokines TNF- $\alpha$, IL-6, Arg1, and IL-10 was assessed ( $n=3$ independent experiments, 10 mice per experiment). All scale bars in a, b $20 \mu \mathrm{m}$. ${ }^{* *} P<0.01$. AR adrenergic receptor, $\alpha 7 \mathrm{nAChR} \alpha-7$ nicotinic acetylcholine receptor, SSC side scatter, ISO isoproterenol, Arg1 arginase 1

than in the control group at some of the time points (Fig. 5c; factorial-design ANOVA, $P<0.01$ at 18 and $24 \mathrm{~h}$ ). In contrast, the number of neutrophils was significantly higher in the mecamylamine-treated group than in the controls, though the timing of their peaks was similar (Fig. 5c; factorial-design ANOVA, $P<0.01)$. These results suggest that the stimulation of nAChRs inhibits the rate and number of neutrophils trafficking to the injured cornea following corneal abrasion.
We also observed the effects of pilocarpine (an mAChR agonist) and atropine (an mAChR antagonist) on the dynamics of neutrophil trafficking to the wounded cornea. We found that, although the number of neutrophils in the pilocarpine group differed significantly from the controls at certain time points, the differences were very small (Fig. $5 \mathrm{~d}$; factorial-design ANOVA, $P<0.01$ at 12,18 , and $24 \mathrm{~h}$ ). In contrast, the number of neutrophils in the atropine-treated group was much higher than in the 
controls (Fig. 5d; factorial-design ANOVA, $P<0.01$ ). These findings suggest that the blockage of $\mathrm{mAChRs}$ with atropine triggers neutrophil influx into the wounded cornea after abrasion.

The ANS influences the production of inflammation-related cytokines following corneal abrasion

Cytokines relevant to inflammation play an important role in wound repair. We examined whether ANS-biased activation influenced a local release of inflammation-related cytokines after corneal abrasion. An enzyme-linked immunosorbent assay (ELISA) on extracts from a pool of 10 corneas collected every $6 \mathrm{~h}$ after epithelial abrasion was performed. Based on our previous report showing that corneal abrasion stimulates a strong immune response by $18 \mathrm{~h}$, we extracted protein from the corneas of different treatment groups at $0,6,12$, and $18 \mathrm{~h}$ postabrasion. ${ }^{5}$ The results revealed striking differences in the production of three murine neutrophil-chemoattractant chemokines: IL-1a, LIX, and MIP-1a. The level of each of these inflammatory cytokines was significantly lower when sympathetic activity was blocked (Fig. 6a, SCGx and timolol groups; factorial-design ANOVA, $P<0.01$ ). In contrast, the levels of IL-1a and MIP-1a were significantly upregulated at 12 and $18 \mathrm{~h}$ after abrasion following the inhibition of parasympathetic activity with the $\mathrm{nAChR}$ antagonist mecamylamine, and the levels of LIX were significantly upregulated $18 \mathrm{~h}$ after abrasion only (Fig. 6b; factorial-design ANOVA, $P<0.01$ ). Increases in IL-1a and MIP-1a were also upregulated 12 and $18 \mathrm{~h}$ after abrasion following the inhibition of parasympathetic activity with the $\mathrm{mAChR}$ antagonist atropine (Fig. 6c; factorial-design ANOVA, $P<0.01)$. In a somewhat different pattern, IL-1a and MIP$1 \mathrm{a}$, but not LIX, were significantly downregulated $18 \mathrm{~h}$ postabrasion following parasympathetic excitation with the $\mathrm{nAChR}$ agonist nicotine. IL-1 $a$ and MIP-1a were significantly downregulated at 12 and $18 \mathrm{~h}$ postabrasion, respectively, with the $\mathrm{mAChR}$ agonist pilocarpine (Fig. 6b, c; factorial-design ANOVA, $P<0.01$ ). Additionally, we compared dynamic changes in the local levels of the proinflammatory cytokine IL- 6 and the anti-inflammatory cytokine IL-10 in ISO- and nicotine-treated groups. The IL- 6 concentration peaked $6 \mathrm{~h}$ after corneal injury in the ISO group, while the IL-10 concentration remained significantly higher 6 and $12 \mathrm{~h}$ after injury in the nicotine group (Fig. $6 \mathrm{~d}$; factorial-design ANOVA, $P<0.01$ ). These results suggest that the ANS is able to promptly regulate the levels of several inflammatory mediators after corneal abrasion. More specifically, our findings imply that sympathetic activation might be involved in triggering the inflammatory response, while parasympathetic activation might be responsible for inhibiting inflammation.

Two distinct subsets of corneal macrophages with different ANS receptor expression

To understand the cellular localization of ANS receptors in the mouse cornea, we employed corneal whole-mount tissue staining and immunofluorescence. The results showed that most of the $\beta 2 A R-p o s i t i v e$ cells costained with the macrophagespecific marker CD64 were found around blood vessels in the limbus (Fig. 7a). Similarly, most of the $\mathrm{CD}^{+} 4^{+}$macrophages around the corneal limbus were also positive for a7nAChR (Fig. 7b). These data suggest that $\mathrm{CD}^{+} 4^{+}$macrophages around the corneal limbus express both $\beta 2 A R$ and $a 7 n A C h R$. To compare the differences in the expression of $\beta 2 A R$ and $a 7 n A C h R$ between these macrophage subsets, we sorted and separated cells and detected the transcriptional profiles of adrenergic and $\mathrm{ACh}$ receptors. As speculated, $\mathrm{CCR}^{+}$cells predominately expressed high levels of Adrb2 (encoding $\beta 2 A R$ ), while CCR2 macrophages predominately expressed high levels of Chrna7 (encoding a7nAChR; Fig. 7c, d). Thus the cornea contains two distinct subsets of macrophages with specific patterns of expression for receptors involved in ANS neurotransmitters: $\mathrm{B2AR}^{\text {high }} \mathrm{CCR}^{+}$and $\mathrm{a} 7 \mathrm{nAChR}{ }^{\text {high }} \mathrm{CCR} 2^{-}$.
Receptors for ANS neurotransmitters mediate the polarization of corneal macrophage activation

The above observations indicate an intratissue specialization among corneal macrophages with the potential for modified responses when the activation of the ANS is biased. We next asked whether $\beta 2 A R$-mediated signaling contributes to in vivo CCR2 ${ }^{+}$ macrophage polarization after topical ISO administration. CCR2 ${ }^{+}$ macrophages were sorted from corneal wounds $6 \mathrm{~h}$ after injury in topically epinephrine-stressed mice, and the gene expression of M1-related proinflammatory cytokines (TNF- $a$ and IL-6) was then determined using qRT-PCR analysis. The results show that treatment with topical epinephrine resulted in significant further upregulation of TNF-a and IL-6 (Fig. 7e, top; factorial-design ANOVA, $P<0.01)$. These data support a crucial role for resident $\mathrm{CCR}^{+}$macrophages in producing the proinflammatory molecules TNF- $\alpha$ and IL- 6 via $\beta 2 A R$ activation in response to local and systemic elevations in epinephrine.

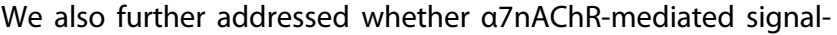
ing contributes to in vivo $\mathrm{CCR}^{-}$macrophage polarization after the topical administration of nicotine on wounded corneas. An analysis of sorted $\mathrm{CCR}^{-}$macrophages from nicotine-treated mice confirmed significantly higher levels of active translation for the M2-related genes IL-10 and Arg1 than were found in sorted macrophages from normal, injured, or locally ISO-treated corneas (Fig. 7e, bottom; factorial-design ANOVA, $P<0.01$ ). These data provide evidence that PSNS activation may trigger changes in $I L$ 10 and $A r g 1$ gene expression in corneal $\mathrm{CCR}^{-}$macrophages via local a7 cholinergic signaling.

\section{DISCUSSION}

We have shown that the cornea is under dual control by the sympathetic and parasympathetic systems, which counterregulate wound repair and inflammation after a corneal injury. Two different macrophage subsets in the cornea preferentially express different ANS neurotransmitter receptors, with the CCR2 ${ }^{+}$ subset expressing the $\beta 2 A R$ and the $C C R 2^{-}$subset expressing a7nAChRs. The pharmacological blockage of $\beta A R$ signaling and the activation of $n A C h R$ signaling accelerated the wound-healing process and inhibited inflammatory responses after corneal abrasion.

Role of sympathetic innervation in the regulation of corneal reepithelialization and proliferation

Through the production of nutrients and trophic factors, sensory nerve fibers play a vital role in maintaining the homeostasis of the corneal epithelium. ${ }^{15}$ However, little is known about the role of autonomic innervation in the corneal epithelium after an injury. Our results revealed that removing SNS activity and blocking the $\beta A R$ signaling pathway not only significantly accelerated reepithelialization of the injured cornea but also shifted the time of peak corneal epithelial mitosis $6-12 \mathrm{~h}$ earlier. This is consistent with studies conducted in vitro in corneal cell culture models using $\beta A R$ antagonists. $^{51,52} \beta A R$ blockage has the potential to accelerate wound closure by altering the epithelial cell shape and cytoskeletal orientation. Indeed, we found that the reverse was also true; topical $\beta A R$ agonists significantly inhibited epithelial migration and proliferation in vivo. Together, these results suggest that sympathetic neurons serve as negative regulators of epithelial cell migration and proliferation after corneal trauma. Conversely, blocking the $\beta A R$ signaling pathway has clinical potential for alleviating impaired wound healing, such as that found in diabetes patients.

Role of parasympathetic innervation in corneal reepithelialization and proliferation

We found that nicotine significantly accelerated reepithelialization and proliferation after a corneal epithelial injury. Studies have 

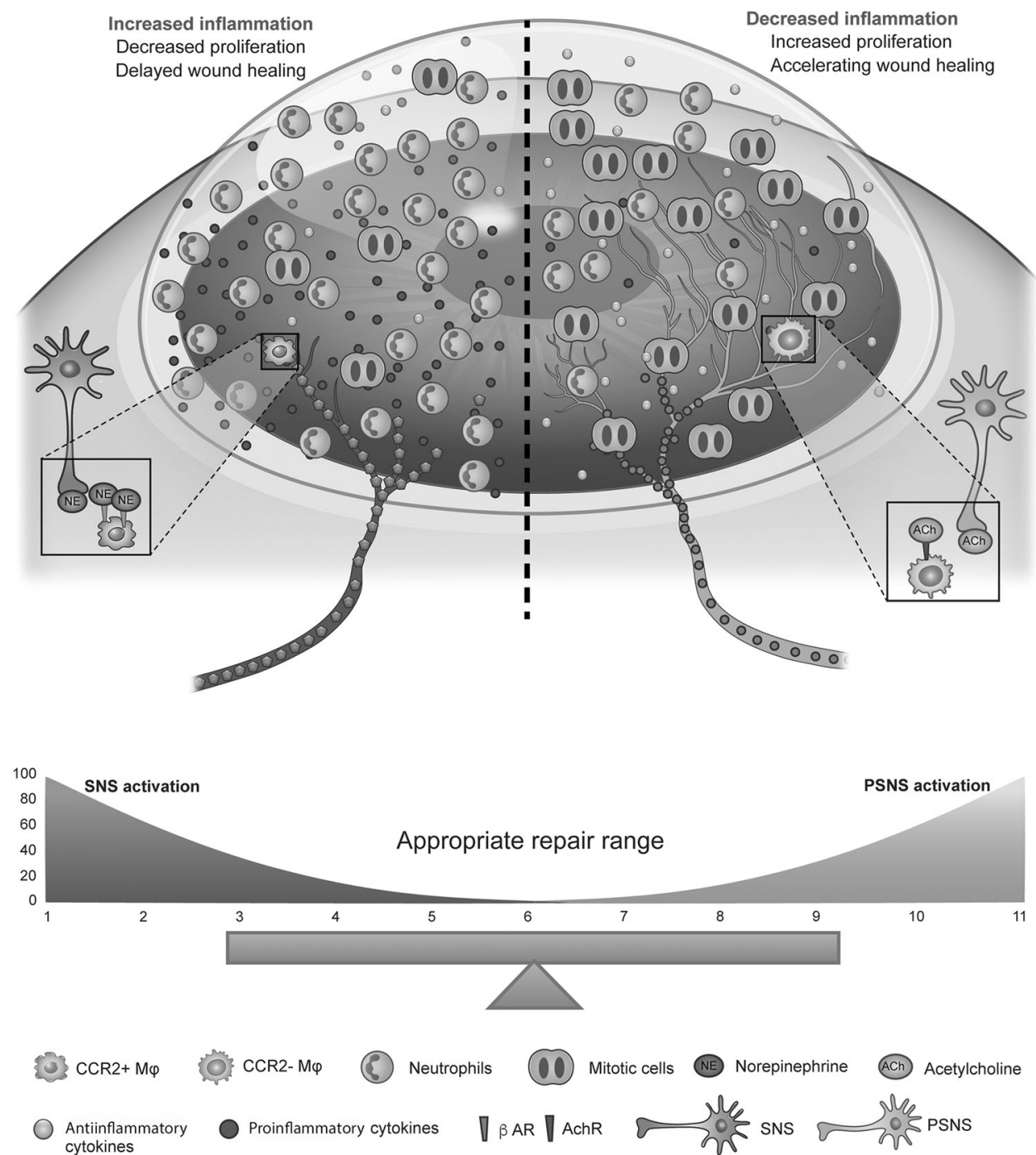

Fig. 8 Schematic illustration showing how corneal wound healing and inflammation might be regulated by dual neural mechanisms. (Left) Nerve endings from the SNS release norepinephrine (NE), which (1) acts on $\beta$-adrenergic receptors ( $\beta$-AR) expressed on corneal cells, where it inhibits the mitosis and migration of epithelial cells; and (2) acts on CCR2 ${ }^{+}$macrophages, where it promotes neutrophil recruitment via the production of neutrophil chemoattractants (IL-1 $\alpha$, IL-6, LIX, TNF- $\alpha$, and MIP-1 $\alpha$ ). (Right) Nerve endings from the PSNS release acetylcholine (ACh), which (1) acts on nicotinergic or/and muscarinic receptors on corneal cells, where it stimulates the proliferation and migration of epithelial cells; and (2) acts on CCR2 ${ }^{-}$macrophages to inhibit inflammation through the production of IL-10 and Arg1. SNS sympathetic nervous system, NE norepinephrine, $\beta$-AR $\beta$-adrenoceptor, PSNS parasympathetic nervous system, ACh acetylcholine, AChR acetylcholine receptor, CCR CC chemokine receptor, IL interleukin, LIX a murine neutrophil-chemoattractant chemokine, TNF tumor necrosis factor, MIP macrophage inflammatory protein, Arg1 arginase 1

shown that, in addition to playing a role in epithelial wound repair, exogenous ${ }^{53}$ or endogenous ${ }^{54}$ nicotine stimulates endothelial cell migration and proliferation. Likewise, another study has shown that nicotine significantly accelerates the wound-healing process in diabetic mouse skin via a mechanism similar to that of the basic fibroblast growth factor. ${ }^{55}$ We found that the use of the topical nonselective channel blocker mecamylamine significantly inhibited corneal regeneration and repair. Interestingly, although the topical application of the $\mathrm{mAChR}$ agonist pilocarpine did not result in significant reepithelialization, the application of the $\mathrm{mAChR}$ antagonist atropine significantly inhibited the reepithelialization process. These contradictory phenomena may be associated with the inhibition of lacrimal gland function by atropine, which effectively decreases the amount of secreted nutrients and growth factors. ${ }^{5,57}$ Another reasonable explanation is that there was already optimal agonist activity in the abraded cornea so that adding additional agonist would have no effect. Overall, these results suggest that, in the case of the cornea, stimulating the nAChRs - but not the mAChRs - of parasympathetic neurons accelerates reepithelialization and proliferation. Given these observations, we propose that $\mathrm{nAChRs}$ are potential targets for treating impaired wound repair.

Role of sympathetic innervation in corneal inflammation Studies have shown that the sympathetic nerves can negatively or positively modulate the inflammatory response in a contextdependent manner. ${ }^{58-60}$ Our study shows that a simple mechanical abrasion of the corneal epithelium can quickly activate the sympathetic nervous system. Moreover, a surgical sympathectomy or a pharmacological blockage of the $\beta A R$ signaling pathway significantly reduces the migration and recruitment of leukocytes to the limbal vascular network after a corneal abrasion. However, 
both the topical application of the adrenergic agonist and the systemic activation of the SNS with the restraint protocol promote neutrophil migration to the wound, suggesting that in the corneal abrasion model, the presence of sympathetic nerves promotes an inflammatory response. This study expands our current understanding of SNS-mediated impairment of wound healing and provides an important potential target for controlling biased overactivation of the SNS, such as stress that leads to exacerbated inflammation via the blockage of the sympathetic signaling pathway.

Role of parasympathetic innervation during corneal inflammation The PSNS also plays an important role in the development, progression, and regression of inflammation, acting in opposition to the SNS to maintain physiological homeostasis. ${ }^{61,62}$ In this study, we found that the topical application of the $n A C h R$ agonist nicotine significantly reduced the number of neutrophils accumulated in the limbus, as well as the number that migrated to the wound area. In contrast, the topical application of the nAChR antagonist mecamylamine increased both the number of limbal leukocytes and their rate of migration to the injured area. This suggests that parasympathetic activation may play an inhibitory role in the inflammatory response to corneal trauma. This is consistent with previous studies reporting that nicotine inhibits leukocyte recruitment and decreases vascular endothelial cell activity during wound healing ${ }^{55}$ and that mecamylamine promotes leukocyte recruitment and endothelial cell viability in the macula of the retina. ${ }^{53}$ Thus the selective activation of cholinergic receptors may modulate inflammatory responses in the cornea. Based on these findings, we propose that the nAChR might also be a promising molecular target for regulating dysfunctional inflammation after corneal abrasion.

Studies show that $\mathrm{mAChRs}$ are also involved in regulating immune and inflammatory responses. Early functional and binding studies revealed the presence of all five mAChRs (M1-M5) on lymphocytes, macrophages, and dendritic cells. ${ }^{50}$ Moreover, spleen cells in M1/M5 knockout mice produce fewer antibodies, less TNF-a, and less IL- 6 than wild-type mice. ${ }^{63}$ Here we first defined the profile of the mAChR subunit expression on the activated neutrophils in the wounded cornea. We also found that the topical administration of the $\mathrm{mAChR}$ antagonist atropine significantly increased the production of proinflammatory cytokines and neutrophil infiltration into the wounded cornea. This suggests that the activation of mAChRs also inhibits a neutrophil influx to the wounded cornea after corneal abrasion. This might also explain that the impairment of reepithelialization after the topical use of atropine described above is partially due to greater neutrophil infiltration.

Polarization of corneal macrophages is controlled by the ANS ANS innervation in peripheral organs and some tissues is linked to enhanced or attenuated inflammatory responses through the release of different neurotransmitters that bind ARs and AChRs in immune cells. ${ }^{64}$ In the intestines, lamina propria macrophages preferentially express a proinflammatory phenotype compared with muscularis macrophages (MMs), which display a tissueprotective phenotype. Upon luminal bacterial infection, extrinsic sympathetic neurons innervating the gut muscularis swiftly activate MMs via norepinephrine signaling to $\beta 2 A R s$ to further enhance tissue-protective programs. ${ }^{25}$ Our data suggest that epinephrine released by SNS neurons initiates a proinflammatory response by binding to $\beta 2 A R s$ that are highly expressed on corneal $\mathrm{CCR}^{+}$macrophages. It also indicates that the $\mathrm{ACh}$ released by PSNS neurons mitigates the inflammatory state by binding to $\mathrm{a} 7 \mathrm{nAChRs}$ that are highly expressed on corneal CCR2 ${ }^{-}$ macrophages. The greater expression of different ANS receptors by local macrophages might represent a mechanism through which the local inflammatory response is regulated by the preferential targeting of these different cell types. However, other resident immune cells, such as T cells, innate lymphoid cells, ${ }^{65}$ and mast cells in the cornea could also express different ANS receptors. ${ }^{66}$ Thus these immune cells may also be involved in this complicated regulatory process, because local innervation and systemic transmitters from the ANS can directly reach these local cells. Future studies are required in order to confirm this.

In conclusion, our results suggest a novel mechanism for the induction and regulation of inflammation after corneal abrasion: the direct ANS-mediated activation of corneal macrophages by two distinct cellular and molecular mechanisms (Fig. 8). More specifically, we found that sympathetic nerve activation supports the local inflammatory reaction by stimulating $\mathrm{CCR}^{+}$macrophages and inhibits the proliferation and migration of corneal epithelial cells by activating $\beta 2 A R$. Conversely, the activation of parasympathetic neurons inhibits local inflammation by stimulating $\mathrm{CCR}^{-}$macrophages and promotes the proliferation and migration of corneal epithelial cells by activating $a 7 n A C h R$. Therefore, once the key players in the mechanisms underlying the autonomic functions have been pinpointed, therapies targeting these systems may effectively control the inflammation and renewal processes after corneal wounding.

\section{METHODS}

\section{Animals}

Male C57BL/6 mice were purchased from the Experimental Animal Center of Sun Yet-Sen University. All the animal experiments were approved by the Animal Experimental Committee at Jinan University Medical School and performed according to the Association for Research in Vision and Ophthalmology (ARVO) statement on the Use of Animals in Ophthalmic and Vision Research. All the mice used in this study were 8-10-week old and weighed $20-25 \mathrm{~g}$.

Corneal epithelial wound model

The wound model was employed as previously described. ${ }^{10}$ Briefly, a central circular epithelial defect $(2 \mathrm{~mm})$ was made with a golf-club-like blade in the murine cornea. To evaluate wound closure, the fluorescein-stained area was photographed with a stereomicroscope every $6 \mathrm{~h}$ and followed by a digital analysis of the stained images until the wound healed.

\section{Whole-mount immunofluorescence}

Immunostaining of whole-mount corneas was performed as previously described. ${ }^{6}$ Briefly, normal or wounded corneas were routinely excised, fixed, washed, blocked, and permeabilized. To stain sympathetic fibers, corneas were incubated with anti-TH antibody or goat-anti-mouse VMAT2 polyclonal antibody, antineuron-specific $\beta$-tubulin III antibody, and APC Rat Anti-Mouse CD31 antibody overnight at $4{ }^{\circ} \mathrm{C}$. To examine the location and distribution of $\beta 2 A R$ in the corneal limbus, corneas were incubated with fluorescein isothiocyanate (FITC) anti- $\beta 2 A R$ antibodies (1:200), phycoerythrin (PE) anti-mouse CD64 for macrophages, and allophycocyanin (APC) rat anti-mouse CD31 for limbal vessels simultaneously. For staining parasympathetic fibers, corneas were incubated with anti-ChAT antibody, anti- $\beta$-tubulin III antibodies, and APC rat anti-mouse CD31 overnight. To show the spatial relationship between limbal vessels, $a 7 n A C h R+$ cells, macrophages, and corneas were incubated with rabbit anti-mouse a7nAChR-FITC, PE anti-mouse CD64, and APC rat anti-mouse CD31 and were then incubated with FITC AffiniPure donkey anti-rabbit immunoglobulin G. Wounded corneas were incubated with the following labeled antibodies overnight a $4{ }^{\circ} \mathrm{C}$ : anti-mouse Ly6G FITC for neutrophils and anti-mouse CD31-PE or APC for the limbic vessel endothelium. Finally, whole corneas were cut into four quadrants, stretched, and mounted with antifade mounting medium containing 4,6-diamidino-2-phenylindole to assess the 
nuclear morphology and cell division. Details regarding the antibodies can be found in Table S1.

qRT-PCR

The corneal tissue was cut into pieces, placed in Buffer RZ, and smashed using a TissueRuptor as described previously. ${ }^{9}$ Total RNA was extracted from the tissue with the RNAsimple Total RNA Kit, and cDNA was synthesized using the ReverTraAce qPCR RT Kit, following the manufacturer's instructions. Further details are provided in the Supplementary Materials and Methods. The PCR primers used in this study are shown in Table S2.

Flow cytometric immunophenotyping, sorting, and transcript amplification in corneal neutrophils and macrophages

Flow cytometric immunophenotyping, sorting, and transcript amplification of corneal neutrophils and macrophages was performed as described previously. ${ }^{9}$ In brief, corneal cell suspensions were obtained by collagenase type-I digestion and stained with a mixture of antibodies including anti-mouse CD45 antibody conjugated with bv510 and anti-mouse Ly6G conjugated with FITC; the stained corneal cells were sorted by flow cytometry using a BD FACSAria to obtain the $\mathrm{Ly}_{6 \mathrm{G}}{ }^{+}$neutrophils. The antibodies, including anti-mouse CD45 antibody conjugated with FITC, antimouse CD64 conjugated with PE, and anti-mouse CCR2 conjugated with $A P C$, were used to stain the macrophages. The stained corneal cells were sorted by flow cytometry using a BD FACSAria to obtain $\mathrm{CD}_{4} 5^{+} \mathrm{CD} 64^{+} \mathrm{CCR} 2{ }^{-}$and $\mathrm{CD} 45^{+} \mathrm{CD} 64^{+} \mathrm{CCR} 2^{+}$ macrophages. Finally, the REPLI-gWTA Single Cell Kit (Qiagen; no. 150063) was used for transcript amplification of small numbers of neutrophils and macrophages.

Pharmacological autonomic agonism and antagonism

To observe the effects of $\beta 2 A R$ agonists and antagonists on wound healing, some mice received a 5 - $\mu$ l eye drop containing either a $0.05 \mathrm{M}$ solution of the $\beta 2 \mathrm{AR}$ agonist ISO or a $0.01 \mathrm{M}$ solution of the $\beta 2 A R$ blocker timolol in BSS at 0,12 , and $24 \mathrm{~h}$ after wounding. To observe the effect of the $\mathrm{mAChR}$ signaling pathway on corneal wound healing, some mice were topically treated with the $\mathrm{mAChR}$ receptor agonist pilocarpine at $0.02 \mathrm{M}$ or with the $\mathrm{mAChR}$ antagonist atropine at $0.05 \mathrm{M}$, each dissolved in BSS.

Restraint (RST) stress model

To induce stress, a daily physical-restraint stress model was used as previously described. ${ }^{40-42}$ In brief, mice were confined within loosely fitting, well-ventilated $50-\mathrm{ml}$ conical tubes for a period of $12 \mathrm{~h}$ during their active nocturnal cycle. Restraint started at 6:00 P. M. (dark onset) and ended at 6:00 A.M. The restraint was initiated 3 days before wounding. Control mice were deprived of food and water during the same time period but were free to roam their cages.

\section{Superior cervical ganglionectomy}

Mouse SCGxs were performed based on a published technique. ${ }^{67}$ Briefly, under general anesthesia with pentobarbital, a $1-\mathrm{cm}$ incision was made in the disinfected skin of the ventral neck region. The salivary glands were exposed and retracted to expose the underlying muscles. After sectioning the omohyoid muscles and dissecting the common carotid artery, the SCG was identified behind the carotid bifurcations and then gently pulled until their avulsion. In the sham group, only-neck-skin incisions were performed without removing the SCG and were closed with nylon sutures.

Chemical sympathectomy

The 6-OHDA in PBS solution was freshly prepared for each experiment and was used immediately for intraperitoneal injections $(200 \mathrm{mg} / \mathrm{kg}) .^{68}$ Sympathectomy was confirmed in the wholemount corneas by immunofluorescent staining (described above) to identify sympathetic nerves. At day 2 after the injection, the cornea was experimentally injured in accordance with the corneal epithelial wounding model. The control animals received injections of PBS alone.

Quantitative analysis of neutrophil influx and epithelial division To compare the difference in the abundance of neutrophils (Ly6G ${ }^{+}$cells) infiltrating the wound regions among the different groups and at different time points after abrasion, we divided each cornea into five regions as previously described ${ }^{5,69}$ (Figure S2). The three-dimensional images through whole corneal thickness in the limbus (Region 1 in Figure S2), the corneal wound margin (Region 3 in Figure S2), and the corneal wound center (Region 5 in Figure S2) were collected using a DeltaVision Elite microscope (GE Healthcare Bio-Sciences, Pittsburgh, PA) at 40x. Two examiners, who were blinded to the corneal specimens, manually counted the neutrophil number in the whole corneal thickness in a stepwise manner by adjusting the scroll arrow of the dialog box in the opened file by the two examiners who were blinded to the corneal specimens. To compare the relative numbers of dividing cells, the corneas were subdivided into nine fields (Regions 1, 2, 3, 4, 5, 4', 3', 2', and $1^{\prime}$ ) from limbus to limbus ${ }^{9}$ (Figure S2).

Measurement of cytokine/chemokine levels in the wounded corneas

IL-1a, LIX, MIP-1a, IL-6, and IL-10 were analyzed by ELISA (RayBiotech, USA) in the extracts of corneas collected at 0, 6, 12, and $18 \mathrm{~h}$ after abrasion, as previously described. ${ }^{6,10}$ Briefly, 10 corneas from each time point were pooled in $1 \mathrm{ml}$ of RPMI 1640 medium. All the samples were subjected to three freeze-thaw cycles and sonication for $30 \mathrm{~s}$ at $250 \mathrm{~Hz}$. All the homogenates were stored at $-80^{\circ} \mathrm{C}$ until needed. The supernatant was used for ELISA according to the manufacturer's instructions. All the samples at each time point were assayed in triplicate.

Statistical analyses

ANOVA tests were performed using the SPSS 17.0 software (SPSS, Inc., Chicago, IL, USA), and $P$-values $<0.05$ were considered statistically significant. All the data are expressed as the mean \pm standard deviation (SD). The statistical significance for wound closure rates was assessed by two-tailed Student's t-tests. Factorial-design ANOVAs with post hoc Tukey's tests were performed to compare data from independent samples.

\section{ACKNOWLEDGEMENTS}

Research support was provided by the National Natural Science Foundation of China through Grants 81470603 (to Z.L.), 81770962 (to Z.L.), and 81700808 (to Y.X.), by the Medical Science and Technology Research Fund of Guangdong Grant A2017279 (to Y.X.), and by NIH grant (5R01EY018239-08).

\section{AUTHOR CONTRIBUTIONS}

Z.L. and Y.X. conceived and designed the study and wrote the manuscript; Y.X. performed most of the experiments and analyzed the data; J.H., Y.G., C.X., C.L., M.W., J. L., T.F., and Y.Y. conducted animal breeding and sample collection. D.D., H.P., L.R., and C.X. contributed to the statistical analyses. All the authors reviewed the manuscript.

\section{ADDITIONAL INFORMATION}

The online version of this article (https://doi.org/10.1038/s41385-018-0031-6) contains supplementary material, which is available to authorized users.

Competing interests: The authors declare no competing interests.

Publisher's note: Springer Nature remains neutral with regard to jurisdictional claims in published maps and institutional affiliations. 


\section{REFERENCES}

1. Ahmed, F., House, R. J. \& Feldman, B. H. Corneal abrasions and corneal foreign bodies. Prim. Care 42, 363-375 (2015).

2. Channa, R. et al. Epidemiology of eye-related emergency department visits. JAMA Ophthalmol. 134, 312-319 (2016).

3. Aslam, S. A., Sheth, H. G. \& Vaughan, A. J. Emergency management of corneal injuries. Injury 38, 594-597 (2007).

4. Szczotka-Flynn, L. B., Pearlman, E. \& Ghannoum, M. Microbial contamination of contact lenses, lens care solutions, and their accessories: a literature review. Eye Contact Lens 36, 116-129 (2010).

5. Li, Z., Burns, A. R. \& Smith, C. W. Two waves of neutrophil emigration in response to corneal epithelial abrasion: distinct adhesion molecule requirements. Invest. Ophthalmol. Vis. Sci. 47, 1947-1955 (2006).

6. Li, Z., Burns, A. R., Han, L., Rumbaut, R. E. \& Smith, C. W. IL-17 and VEGF are necessary for efficient corneal nerve regeneration. Am. J. Pathol. 178, 1106-1116 (2011).

7. Li, Z., Burns, A. R. \& Smith, C. W. Lymphocyte function-associated antigen-1dependent inhibition of corneal wound healing. Am. J. Pathol. 169, 1590-1600 (2006).

8. Liu, Q., Smith, C. W., Zhang, W., Burns, A. R. \& Li, Z. NK cells modulate the inflammatory response to corneal epithelial abrasion and thereby support wound healing. Am. J. Pathol. 181, 452-462 (2012).

9. Liu, J. et al. CCR2(-) and CCR2(+) corneal macrophages exhibit distinct characteristics and balance inflammatory responses after epithelial abrasion. Mucosal Immunol. 10, 1145-1159 (2017).

10. Li, Z., Burns, A. R., Miller, S. B. \& Smith, C. W. CCL20, gammadelta T cells, and IL-22 in corneal epithelial healing. FASEB J. 25, 2659-2668 (2011).

11. Ebihara, N., Matsuda, A., Nakamura, S., Matsuda, H. \& Murakami, A. Role of the IL-6 classic- and trans-signaling pathways in corneal sterile inflammation and wound healing. Invest. Ophthalmol. Vis. Sci. 52, 8549-8557 (2011).

12. Zhang, W., Magadi, S., Li, Z., Smith, C. W. \& Burns, A. R. IL-20 promotes epithelial healing of the injured mouse cornea. Exp. Eye Res. 154, 22-29 (2017).

13. Bukowiecki, A., Hos, D., Cursiefen, C. \& Eming, S. A. Wound-healing studies in cornea and skin: parallels, differences and opportunities. Int. J. Mol. Sci. 18, 1257 (2017).

14. McDougal, D. H. \& Gamlin, P. D. Autonomic control of the eye. Compr. Physiol. 5, 439-473 (2015).

15. Muller, L. J., Marfurt, C. F., Kruse, F. \& Tervo, T. M. Corneal nerves: structure, contents and function. Exp. Eye Res. 76, 521-542 (2003)

16. Marfurt, C. F. \& Ellis, L. C. Immunohistochemical localization of tyrosine hydroxylase in corneal nerves. J. Comp. Neurol. 336, 517-531 (1993).

17. Marfurt, C. F., Kingsley, R. E. \& Echtenkamp, S. E. Sensory and sympathetic innervation of the mammalian cornea. A retrograde tracing study. Invest. Ophthalmol. Vis. Sci. 30, 461-472 (1989).

18. Marfurt, C. F., Jones, M. A. \& Thrasher, K. Parasympathetic innervation of the rat cornea. Exp. Eye Res. 66, 437-448 (1998).

19. Ivanusic, J. J., Wood, R. J. \& Brock, J. A. Sensory and sympathetic innervation of the mouse and guinea pig corneal epithelium. J. Comp. Neurol. 521, 877-893 (2013).

20. Mahmoud, A. I. et al. Nerves regulate cardiomyocyte proliferation and heart regeneration. Dev. Cell 34, 387-399 (2015).

21. White, I. A., Gordon, J., Balkan, W. \& Hare, J. M. Sympathetic reinnervation is required for mammalian cardiac regeneration. Circ. Res. 117, 990-994 (2015).

22. Nance, D. M. \& Sanders, V. M. Autonomic innervation and regulation of the immune system (1987-2007). Brain Behav. Immun. 21, 736-745 (2007).

23. Bellinger, D. L. \& Lorton, D. Autonomic regulation of cellular immune function. Auton. Neurosci. 182, 15-41 (2014).

24. Scheiermann, $C$. et al. Adrenergic nerves govern circadian leukocyte recruitment to tissues. Immunity 37, 290-301 (2012).

25. Gabanyi, I. et al. Neuro-immune interactions drive tissue programming in intestinal macrophages. Cell 164, 378-391 (2016).

26. Pirzgalska, R. M. et al. Sympathetic neuron-associated macrophages contribute to obesity by importing and metabolizing norepinephrine. Nat. Med. 23, 1309-1318 (2017).

27. Rosas-Ballina, M. et al. Acetylcholine-synthesizing $T$ cells relay neural signals in a vagus nerve circuit. Science 334, 98-101 (2011).

28. Racosta, J. M. \& Kimpinski, K. Autonomic dysfunction, immune regulation, and multiple sclerosis. Clin. Auton. Res. 26, 23-31 (2016).

29. Bernik, T. R. et al. Pharmacological stimulation of the cholinergic antiinflammatory pathway. J. Exp. Med. 195, 781-788 (2002).

30. Tracey, K. J. Physiology and immunology of the cholinergic antiinflammatory pathway. J. Clin. Invest. 117, 289-296 (2007).

31. Wang, $\mathrm{H}$. et al. Nicotinic acetylcholine receptor alpha7 subunit is an essential regulator of inflammation. Nature 421, 384-388 (2003).

32. Wang, $\mathrm{H}$. et al. Cholinergic agonists inhibit HMGB1 release and improve survival in experimental sepsis. Nat. Med. 10, 1216-1221 (2004).
33. de Jonge, W. J. et al. Stimulation of the vagus nerve attenuates macrophage activation by activating the Jak2-STAT3 signaling pathway. Nat. Immunol. 6, 844-851 (2005).

34. Lu, B. et al. alpha7 nicotinic acetylcholine receptor signaling inhibits inflammasome activation by preventing mitochondrial DNA release. Mol. Med. 20, 350-358 (2014).

35. Inoue, T. et al. Vagus nerve stimulation mediates protection from kidney ischemia-reperfusion injury through alpha7nAChR+splenocytes. J. Clin. Invest. 126, 1939-1952 (2016).

36. Hamrah, P., Huq, S. O., Liu, Y., Zhang, Q. \& Dana, M. R. Corneal immunity is mediated by heterogeneous population of antigen-presenting cells. J. Leukoc. Biol. 74, 172-178 (2003).

37. Brissette-Storkus, C. S., Reynolds, S. M., Lepisto, A. J. \& Hendricks, R. L. Identification of a novel macrophage population in the normal mouse corneal stroma. Invest. Ophthalmol. Vis. Sci. 43, 2264-2271 (2002).

38. Souza, B. R., Cardoso, J. F., Amadeu, T. P., Desmouliere, A. \& Costa, A. M. Sympathetic denervation accelerates wound contraction but delays reepithelialization in rats. Wound Repair Regen. 13, 498-505 (2005).

39. Zheng, Z. et al. Sympathetic denervation accelerates wound contraction but inhibits reepithelialization and pericyte proliferation in diabetic mice. J. Diabetes Res. 2017, 7614685 (2017).

40. Gajendrareddy, P. K., Sen, C. K., Horan, M. P. \& Marucha, P. T. Hyperbaric oxygen therapy ameliorates stress-impaired dermal wound healing. Brain Behav. Immun. 19, 217-222 (2005).

41. Padgett, D. A., Marucha, P. T. \& Sheridan, J. F. Restraint stress slows cutaneous wound healing in mice. Brain Behav. Immun. 12, 64-73 (1998).

42. Tymen, S. D. et al. Restraint stress alters neutrophil and macrophage phenotypes during wound healing. Brain Behav. Immun. 28, 207-217 (2013).

43. Kim, M. H. et al. Catecholamine stress alters neutrophil trafficking and impairs wound healing by beta2-adrenergic receptor-mediated upregulation of IL-6. J. Invest. Dermatol. 134, 809-817 (2014).

44. Loftus, T. J., Efron, P. A., Moldawer, L. L. \& Mohr, A. M. Beta-blockade use for traumatic injuries and immunomodulation: a review of proposed mechanisms and clinical evidence. Shock 46, 341-351 (2016).

45. Janig, W. Sympathetic nervous system and inflammation: a conceptual view. Auton. Neurosci. 182, 4-14 (2014).

46. Lorton, D. \& Bellinger, D. L. Molecular mechanisms underlying beta-adrenergic receptor-mediated cross-talk between sympathetic neurons and immune cells. Int. J. Mol. Sci. 16, 5635-5665 (2015).

47. Liu, S., Li, J., Tan, D. T. \& Beuerman, R. W. Expression and function of muscarinic receptor subtypes on human cornea and conjunctiva. Invest. Ophthalmol. Vis. Sci. 48, 2987-2996 (2007).

48. Huston, J. M. et al. Splenectomy inactivates the cholinergic antiinflammatory pathway during lethal endotoxemia and polymicrobial sepsis. J. Exp. Med. 203, 1623-1628 (2006).

49. Leib, C. et al. Role of the cholinergic antiinflammatory pathway in murine autoimmune myocarditis. Circ. Res. 109, 130-140 (2011).

50. Fujii, T. et al. Expression and function of the cholinergic system in immune cells. Front. Immunol. 8, 1085 (2017).

51. Pullar, C. E. et al. Beta-adrenergic receptor agonists delay while antagonists accelerate epithelial wound healing: evidence of an endogenous adrenergic network within the corneal epithelium. J. Cell. Physiol. 211, 261-272 (2007).

52. Ghoghawala, S. Y., Mannis, M. J., Pullar, C. E., Rosenblatt, M. I. \& Isseroff, R. R. Beta2-adrenergic receptor signaling mediates corneal epithelial wound repair. Invest. Ophthalmol. Vis. Sci. 49, 1857-1863 (2008).

53. Campochiaro, P. A. et al. Topical mecamylamine for diabetic macular edema. Am. J. Ophthalmol. 149, 839-851 e831 (2010).

54. Cooke, J. P. \& Ghebremariam, Y. T. Endothelial nicotinic acetylcholine receptors and angiogenesis. Trends Cardiovasc. Med. 18, 247-253 (2008).

55. Jacobi, J. et al. Nicotine accelerates angiogenesis and wound healing in genetically diabetic mice. Am. J. Pathol. 161, 97-104 (2002).

56. Klenkler, B., Sheardown, H. \& Jones, L. Growth factors in the tear film: role in tissue maintenance, wound healing, and ocular pathology. Ocul. Surf. 5, 228-239 (2007).

57. Ji, Y. W. et al. Lacrimal gland-derived IL-22 regulates IL-17-mediated ocular mucosal inflammation. Mucosal Immunol. 10, 1202-1210 (2017).

58. Grebe, K. M. et al. Sympathetic nervous system control of anti-influenza CD8+T cell responses. Proc. Natl. Acad. Sci. USA 106, 5300-5305 (2009).

59. Grebe, K. M. et al. Cutting edge: sympathetic nervous system increases proinflammatory cytokines and exacerbates influenza A virus pathogenesis. J. Immunol. 184, 540-544 (2010).

60. Prass, K. et al. Stroke-induced immunodeficiency promotes spontaneous bacterial infections and is mediated by sympathetic activation reversal by poststroke $T$ helper cell type 1-like immunostimulation. J. Exp. Med. 198, 725-736 (2003). 
61. Olofsson, P. S., Rosas-Ballina, M., Levine, Y. A. \& Tracey, K. J. Rethinking inflammation: neural circuits in the regulation of immunity. Immunol. Rev. 248, 188-204 (2012).

62. Goehler, L. E. et al. Vagal immune-to-brain communication: a visceral chemosensory pathway. Auton. Neurosci. 85, 49-59 (2000).

63. Fujii, Y. X. et al. Diminished antigen-specific $\lg G 1$ and interleukin-6 production and acetylcholinesterase expression in combined M1 and M5 muscarinic acetylcholine receptor knockout mice. J. Neuroimmunol. 188, 80-85 (2007).

64. Veiga-Fernandes, H. \& Mucida, D. Neuro-immune interactions at barrier surfaces. Cell 165, 801-811 (2016).

65. Galle-Treger, L. et al. Nicotinic acetylcholine receptor agonist attenuates ILC2dependent airway hyperreactivity. Nat. Commun. 7, 13202 (2016).

66. Chavan, S. S. \& Tracey, K. J. Essential neuroscience in immunology. J. Immunol. 198, 3389-3397 (2017).

67. Savastano, L. E. et al. A standardized surgical technique for rat superior cervical ganglionectomy. J. Neurosci. Methods 192, 22-33 (2010).

68. Bhowmick, S. et al. The sympathetic nervous system modulates CD4(+)FoxP3(+) regulatory T cells via a TGF-beta-dependent mechanism. J. Leukoc. Biol. 86, 1275-1283 (2009).
69. Wang, H. et al. Epothilone B speeds corneal nerve regrowth and functional recovery through microtubule stabilization and increased nerve beading. Sci. Rep. 8, 2647 (2018).

(i) Open Access This article is licensed under a Creative Commons Attribution 4.0 International License, which permits use, sharing, adaptation, distribution and reproduction in any medium or format, as long as you give appropriate credit to the original author(s) and the source, provide a link to the Creative Commons license, and indicate if changes were made. The images or other third party material in this article are included in the article's Creative Commons license, unless indicated otherwise in a credit line to the material. If material is not included in the article's Creative Commons license and your intended use is not permitted by statutory regulation or exceeds the permitted use, you will need to obtain permission directly from the copyright holder. To view a copy of this license, visit http://creativecommons. org/licenses/by/4.0/.

(c) The Author(s) 2018 